\title{
Skeletal-level control-based forward dynamic analysis of acquired healthy and assisted gait motion
}

\author{
F. Mouzo ${ }^{1}$, U. Lugris ${ }^{1}$, R. Pamies-Vila ${ }^{2}$, J. Cuadrado $^{1}$ \\ ${ }^{1}$ Escuela Politecnica Superior, University of La Coruña, javier.cuadrado@udc.es \\ 2 Dept. of Mechanical Engineering, Universitat Politecnica de Catalunya, \\ rosa.pamies@upc.edu
}

Keywords: multibody dynamics, human models, gait simulation, forward dynamics, underactuated systems, contact models, assistive devices

\begin{abstract}
Gait analysis is commonly addressed through inverse dynamics. However, forward dynamics can be advantageous when descending to muscular level, as it allows that activation and contraction equations are integrated with motion thus providing better dynamic consistency, or when studying assisted gait, as it enables the estimation of the interaction forces between subject and devices even if the motion capture process doesn't provide enough resolution to distinguish the motions of limb and device. Control-based methods seem to be the most natural choice to carry out the forward-dynamics analysis of an acquired gait, but several options exist in their application. The paper explores such options for healthy and assisted gait, and concludes that the computed torque control of all the subject's degrees of freedom is the alternative that provides the most accurate results. Moreover, the study of its more problematic underactuated variant accompanied by contact models showed to be connected to neighbor challenging topics as gait prediction or walking simulation of humanoids.
\end{abstract}

\section{Introduction}

The use of multibody models of humans has become more and more popular in the last decades. Basically, the objective can be to analyze the real motion performed by a subject [1] or to predict the motion that a subject would develop under certain conditions [2]. Both objectives are of great relevance for medical, sport and ergonomical applications, among others. Being less difficult, the analytic objective was addressed first, while its predictive counterpart has received attention in the present century. On the other hand, gait is possibly the human motion that has attracted the most interest among researchers, as it represents a key capability in healthy subjects, while its alteration or elimination due to illness or accident causes a relevant reduction in the quality of life of the affected person. This paper addresses the topic of carrying out gait analysis by means of multibody human models.

Usually, to perform gait analysis, a more or less accurate multibody model of the subject is built. This model is then animated using the real motion, optically captured during an experiment. Afterwards, as the motion of the model is known, an inverse-dynamics analysis is applied to solve for the external reactions and the net joint torques that generated the motion. This approach works perfectly for the single-support phase of gait, since the only external reactions are the three components of force and the three components of moment due to the foot-ground contact, i.e. six reactions in all. However, during the double-support phase, the external reactions increase to twelve, as the two feet are in contact with the ground, thus leading to an indeterminacy. To overcome this 
problem, the foot-ground contact reactions are typically measured during the experiment by means of force plates, and introduced in the equations of motion, so that only the net joint torques are kept as unknowns. Unfortunately, due to the various sources of error in both the experimental and computational processes, some discrepancies are usually found between the total external reactions yielded by the inverse dynamic analysis and those experimentally measured, which leads to some inconsistency in the results. To mitigate that, many solutions have been proposed in the literature which basically modify the acquired motion or the model parameters so that the mentioned discrepancies are minimized [3]. This inverse-dynamics based method of gait analysis at skeletal level is usually accompanied, at musculo-skeletal level, by static optimization methods to estimate muscle forces [4].

An alternative way of addressing gait analysis is by means of forward-dynamics analysis. This may sound strange, since motion is known and, hence, the obvious approach to solve for the external reactions and net joint torques would be inverse dynamics. However, the problem can be arranged in such a way that the equations of motion are integrated with respect to time. This option can be advantageous in some cases. For example, when descending to muscular level, the forward-dynamics based solution enables muscle activation and contraction equations to be integrated in time along with the equations of motion [5]. Although activation, or even activation and contraction dynamics, can also be considered through inverse-dynamics based approaches [6-9] to avoid physiologically impossible histories of the muscle forces, an ulterior simulation having as inputs the obtained excitation histories would drift from the captured motion in unstable cases like gait. Also, when the subject employs assistive devices to walk, as orthoses or exoskeletons, a forward-dynamics approach makes it possible to separately consider the dynamics of the subject and of the assistive devices, thus enabling the estimation of subject-device interaction forces when the motion capture process doesn't provide enough resolution to distinguish the motions of limb and device in order to apply an inverse-dynamics based approach [10]. However, some disadvantages arise too when the forward-dynamics approach is applied: since it implies the time integration of the model equations of motion, the inherent challenges of gait dynamics (intermittent contact, stability, etc.) must be faced. In fact, the problem has much in common with the simulation of walking humanoids [11], and may even be perceived as an intermediate step towards human gait prediction [12], with a lower level of difficulty as the resulting motion is known beforehand.

Some works can be found in the literature that use a forward-dynamics approach for gait analysis. For example, in [5] the computed muscle control (CMC) method for gait analysis at neuro-musculo-skeletal level is presented: at the skeletal stage, the method applies the computed torque control (CTC) method to the muscle-actuated degrees of freedom of the model, while the six degrees of freedom of the base body are governed by a kind of proportional-derivative controllers with variable gains acting at the feet. Another example is [10], where the interaction forces between the subject's limb and an ankle-foot orthosis are sought to be estimated: most subject's degrees of freedom (except the ankle angle which is left to its own dynamics) are kinematically guided, as is the orthotic ankle motion. Summarizing, in previous contributions several strategies are present, such as controllers of different natures aimed at tracking joint trajectories or base body motion, kinematic guidance of some degrees of freedom of the model, and allowance of free motion for others. 
If the motion is known and the model dynamic equations have to be integrated in time as required by the forward-dynamics approach, the most obvious procedure seems to be the application of control-based methods that seek to track the acquired gait. Imposing the acquired value to some model coordinates cannot be considered forward dynamics, while kinematic guidance through rheonomic constraints could be equivalent to control-based methods, depending on the method used for constraint enforcement. However, such a control scheme can be applied to all the degrees of freedom in the subject's model, or just to those corresponding to the joints (the ones that can actually be controlled by the subject) while the six degrees of freedom of the base body receive the measured ground reactions, either directly or through contact models. Hence, there are several options that must be studied, in order to clarify which is the most appropriate method to conduct the forward-dynamics analysis of an acquired gait, for the cases when it proves to be advantageous with respect to the inverse-dynamics approach. Therefore, the objective of this paper is to explore, in a systematic and consistent way, the available control-based options to carry out the forward-dynamics approach for both healthy and assisted gait analysis at skeletal level, and to give some criteria about their use.

The remaining of the paper is organized as follows. Section 2 describes the experiments of healthy gait along with the planar and three-dimensional models that were developed for the study. In Sections 3 and 4, respectively, the multibody formulation used and its application to the inverse-dynamics analysis of gait are briefly described. Section 5 addresses the forward-dynamics analysis of healthy gait in the case of full actuation, in which all the degrees of freedom of the model are controlled. In Section 6, only the joint degrees of freedom are controlled (underactuation), while several options to account for the ground reactions are considered. The strategies adopted in Sections 5 and 6 are applied to assisted gait in Section 7 and, finally, Section 8 presents the discussion of the results obtained in the three preceding sections and gathers the conclusions of the work.

\section{Experiments and models}

In this paper two models for healthy gait were used, one planar and another one threedimensional. Although the three-dimensional model was used in all the cases along the paper, its planar counterpart was useful when addressing for the first time the most challenging approach, i.e. the underactuated model with ground reactions from a contact model. Both models correspond to the same subject and experiment. The 3D model presented here will be further complemented in Section 7 for assisted gait.

\subsection{Three-dimensional model}

A three dimensional model was created by the authors to represent a healthy adult male, 27 years old, mass $84 \mathrm{~kg}$ and height $1.75 \mathrm{~m}$, who was selected to carry out the following experiment. The subject walked on a walkway featuring two embedded force plates (AMTI, AccuGait sampling at $100 \mathrm{~Hz}$ ), and his motion was captured by 12 optical infrared cameras (Natural Point, OptiTrack FLEX:V100 also sampling at $100 \mathrm{~Hz}$ ) that computed the position of 37 optical markers. The study was approved by the institutional ethical committee and the subject gave his informed consent.

The human body was modeled as a 3D multibody system formed by rigid bodies, as shown in Fig. 1. It consists of 18 anatomical segments: two hindfeet, two forefeet, two shanks, two thighs, pelvis, torso, neck, head, two arms, two forearms and two hands. The 
segments are linked by ideal spherical joints thus defining a model with 57 degrees of freedom. The global axes were defined as follows: $x$-axis in the postero-anterior direction, $y$-axis in the medio-lateral direction, and $z$-axis in the vertical direction. The computational model was defined with 228 mixed (natural and angular) coordinates. The subset of natural coordinates comprises the three Cartesian coordinates of 22 points, and the three Cartesian components of 36 unit vectors, thus making a total of 174 variables. The points correspond to the positions of all the joints (white dots in Fig. 1), along with points of the five distal segments -head, hands and forefeet- (black dots in Fig. 1). Each one of the 18 bodies was defined by its proximal and distal points, plus two orthogonal unit vectors aligned at the postero-anterior and medio-lateral directions, respectively (red and green vectors in Fig. 1), when the model is in the standing posture. The remaining 54 variables were the 18 sets of 3 angles that define the orientation of each body with respect to the inertial frame.

The geometric and inertial parameters of the model were obtained, for the lower limbs, by applying regression equations from a reduced set of measurements taken on the subject, following the procedures described in [13]. For the upper part of the body, data from standard tables [14] were scaled according to the mass and height of the subject. In order to adjust the total mass of the subject, a second scaling was applied to the inertial parameters of the upper part of the body.

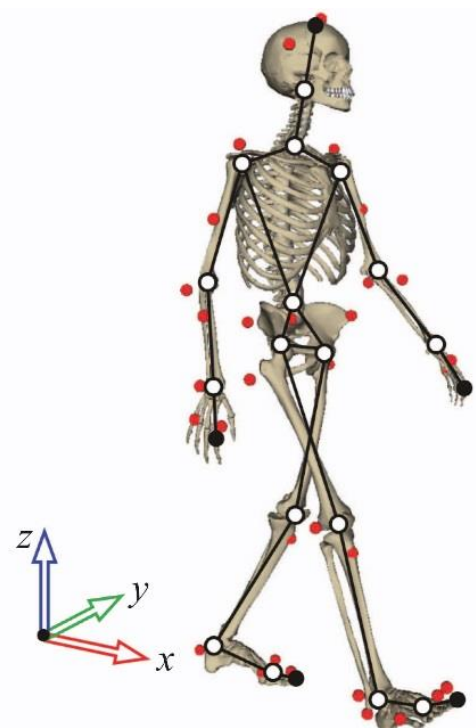

(a)

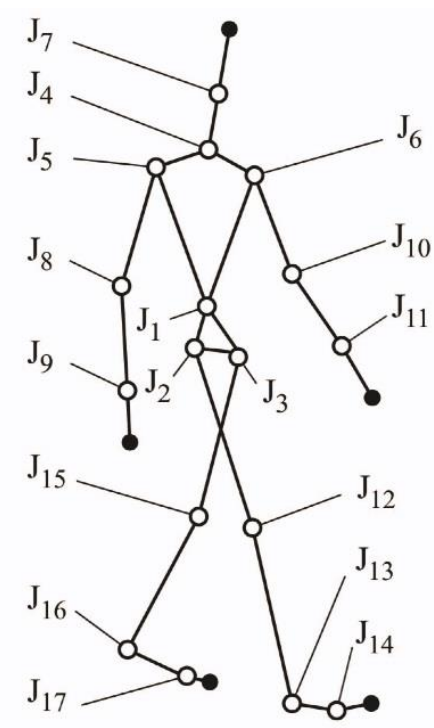

(b)

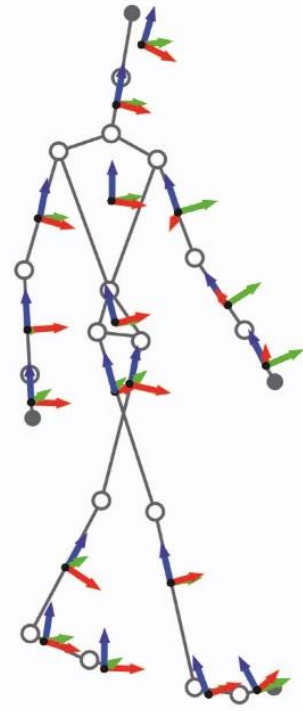

(c)

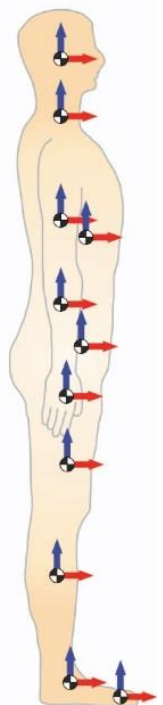

(d)

Figure 1. Three-dimensional model.

The kinematic information of the motion was obtained from the trajectories of the 37 markers attached to the subject's body (red dots in Fig. 1a), which were captured at 100 $\mathrm{Hz}$ frequency by means of the 12 infrared cameras. Position data were filtered using an algorithm based on Singular Spectrum Analysis (SSA) and the natural coordinates of the model were calculated using algebraic relations. Afterwards, a minimization procedure ensured the kinematic consistency of the natural coordinates. From that information, the histories of a set of 57 independent coordinates -as many as the system degrees of freedom-formed by the Cartesian coordinates of the position vector of the lumbar joint $\left(\mathrm{J}_{1}\right.$ in Fig. 1b) and the $18 \times 3$ angles that define the absolute orientation of each body, were kinematically obtained and approximated by B-spline curves. Analytical 
differentiation yielded the corresponding velocity and acceleration histories. More detail about the treatment of the captured data can be found in [15].

\subsection{Planar model}

The planar model was obtained as the projection on the sagital plane of a simplifed version of the three-dimensional model described before. The model features 12 segments and 14 degrees of freedom, as shown in Fig. 2. The global axes were defined as follows: $x$-axis in the postero-anterior direction, $y$-axis in the vertical direction. The computational model was defined with 38 mixed (natural and angular) coordinates. The subset of natural coordinates comprises the two Cartesian coordinates of 13 points, which means 26 variables. The points correspond to the positions of all the revolute joints (white dots in Fig. 2 left), along with points of the five distal segments -head, forearms and feet- (black dots in Fig. 2 left). Each one of the 12 bodies was defined by its proximal and distal points. The remaining 12 variables are the angle that defines the orientation of the trunk with respect to the $y$-axis of the inertial frame, plus the 11 angles that define the orientation of each segment with respect to the previous one in the kinematic chain (Fig. 2 right).
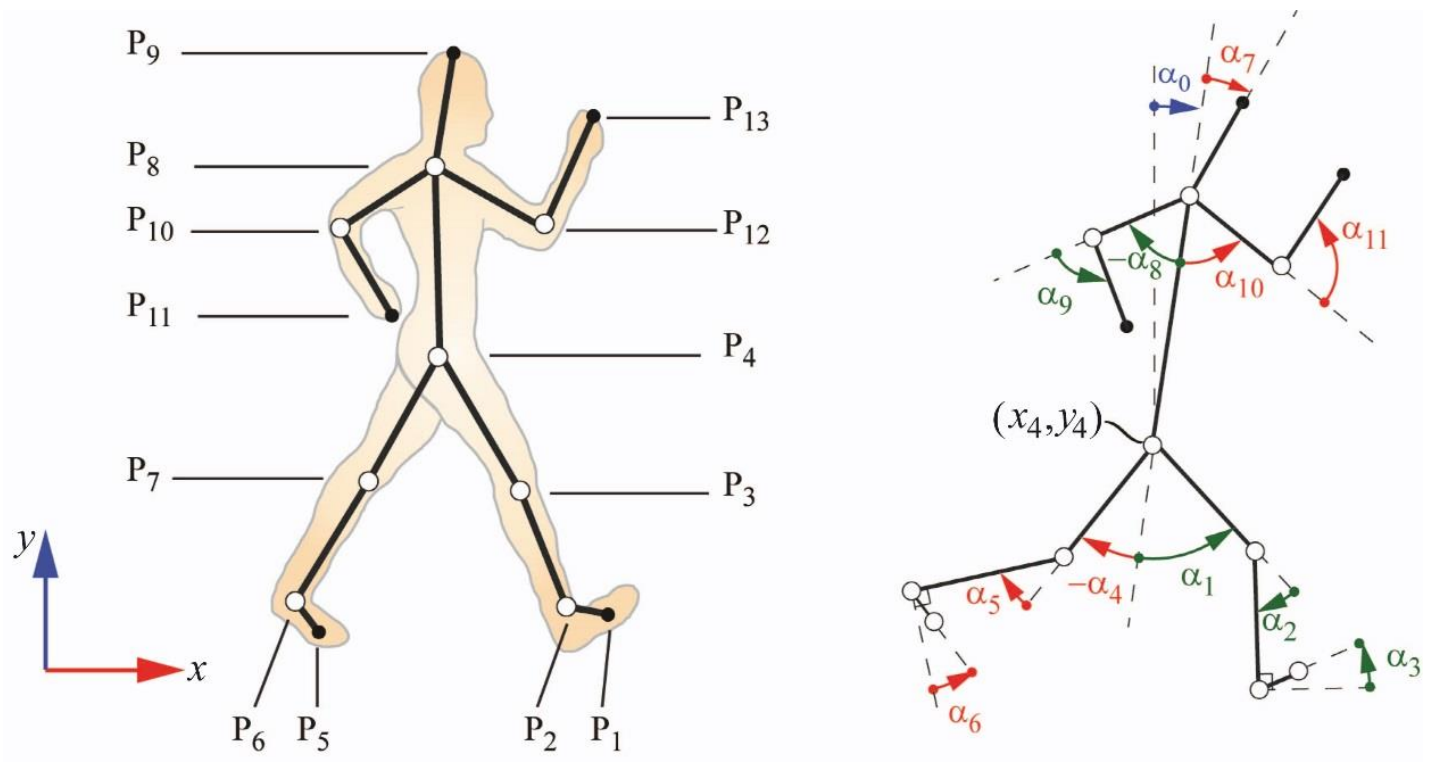

Figure 2. Planar model.

This planar model can be found in the Library of Computational Benchmark Problems [16] developed by the IFToMM Technical Committee for Multibody Dynamics. The benchmark problem, named Gait 2D, provides the geometrical and inertial parameters of the model, the histories of the markers used to optically capture the motion of the subject described above, and the ground reactions measured by force plates. The temporal evolution of the 14 independent coordinates defining the motion, i.e. the two Cartesian coordinates of the hip joint and the 12 angular variables shown in Fig. 2 right, obtained after the same processing as that explained for the 3D model, are supplied too.

\section{Multibody formulation}

In this work, the formulation in minimum number of coordinates proposed in [17] and called matrix-R formulation was used. In this formulation, two sets of coordinates are 
considered: a set of dependent coordinates, $\mathbf{q}$, and a set of independent coordinates, $\mathbf{z}$. In the $3 \mathrm{D}$ model, vector $\mathbf{q}$ is formed by the 228 model coordinates, while vector $\mathbf{z}$, of size 57, is composed by the three Cartesian coordinates of the lumbar joint plus the 18 sets of three angles defining the orientation of the bodies. In the $2 \mathrm{D}$ model, vector $\mathbf{q}$ is formed by the 38 model coordinates, while vector $\mathbf{z}$, of size 14 , is composed by the two Cartesian coordinates of the hip joint plus the 12 angles shown in Fig. 2 right. The equations of motion are stated as,

$$
\mathbf{R}^{\mathrm{T}} \overline{\mathbf{M}} \mathbf{R} \ddot{\mathbf{z}}=\mathbf{R}^{\mathrm{T}}(\overline{\mathbf{Q}}-\overline{\mathbf{M}} \dot{\mathbf{R}} \dot{\mathbf{z}})
$$

where $\overline{\mathbf{M}}$ is the mass matrix referred to $\mathbf{q}, \mathbf{R}$ is the matrix relating the dependent and independent velocities, $\dot{\mathbf{q}}=\mathbf{R} \dot{\mathbf{z}}$, and $\overline{\mathbf{Q}}$ is the vector of generalized forces referred to $\mathbf{q}$. Therefore, the equations of motion (1) can be written in a compact form as,

$$
\mathbf{M} \ddot{\mathbf{z}}=\mathbf{Q}
$$

with $\mathbf{M}=\mathbf{R}^{\mathrm{T}} \overline{\mathbf{M}} \mathbf{R}$ and $\mathbf{Q}=\mathbf{R}^{\mathrm{T}}(\overline{\mathbf{Q}}-\overline{\mathbf{M}} \dot{\mathbf{R}} \dot{\mathbf{z}})$ the mass matrix and vector of generalized forces referred to $\mathbf{z}$, respectively. The number of equations in (2) is 57 in the 3D case and 14 in the $2 \mathrm{D}$ case, as many as the number of degrees of freedom of the model.

For the forward-dynamics analysis, the equations of motion (2) were numerically integrated in time by means of the single step, fixed time step, trapezoidal rule.

\section{Inverse dynamic analysis}

First of all, an inverse-dynamics analysis (IDA) of the experimentally measured motion was performed (both for the 2D and 3D cases). As explained before, the histories of positions, velocities and accelerations of the independent coordinates, $\mathbf{z}, \dot{\mathbf{z}}, \ddot{\mathbf{z}}$, and of the dependent coordinates, $\mathbf{q}, \dot{\mathbf{q}}, \ddot{\mathbf{q}}$, were already known from the processing of the motion capture data. Then, writing the equations of motion (2) as,

$$
\mathbf{M} \ddot{\mathbf{z}}=\mathbf{Q}_{\mathrm{m}}+\mathbf{Q}_{\mathbf{r}}
$$

where $\mathbf{Q}_{\mathrm{m}}$ is the vector of generalized motor forces and $\mathbf{Q}_{\mathbf{r}}$ is the vector grouping all the remaining generalized forces, the unknown vector $\mathbf{Q}_{\mathrm{m}}$ can be obtained as,

$$
\mathbf{Q}_{\mathrm{m}}=\mathbf{M} \ddot{\mathbf{z}}-\mathbf{Q}_{\mathrm{r}}
$$

which provides the net joint torques and the ground reactions that produced the acquired motion.

The obtained ground reactions and net joint torques correspond to considering the pelvis (or trunk in the 2D case) as the base body. They can easily be transformed to their counterparts when the supporting foot is considered as the base body, thus yielding the proper values of external reactions and net joint torques. However, when the two feet are contacting the ground, the external reactions must be distributed between them. In this 
work, this was done in the same proportion shown by the reactions measured by means of force plates, following the approach presented in [15]. Therefore, the computed ground reactions are fully consistent with the motion, and no residual wrenches are present.

\section{Forward dynamics: fully actuated system}

In this work, the first approach to carry out a forward-dynamics analysis (FDA) of the acquired gait motion consisted of using trajectory tracking controllers associated to all the system degrees of freedom. This implies assuming that there exist actuators not only at joint level, which is indeed the case, but also for the six degrees of freedom of the base body (in the 3D case), which does not obviously correspond to reality. In fact, these external inputs come from the ground reactions, which are not modeled when using the present approach. They will appear in the next Section.

The 3D model described before was used for this Section. The equations of motion (2) are written again as,

$$
\mathbf{M} \ddot{\mathbf{z}}=\mathbf{Q}_{\mathrm{u}}+\mathbf{Q}_{\mathrm{r}}
$$

where $\mathbf{Q}_{\mathrm{u}}$ is the vector of the inputs provided by the controllers and $\mathbf{Q}_{\mathbf{r}}$ is the vector of the remaining generalized forces. Three methods were considered within this first approach.

\subsection{Computed feedforward}

In the first method, the actuators associated to the system degrees of freedom introduce as inputs the external reactions and net joint torques previously calculated through inverse dynamics, so that,

$$
\mathbf{Q}_{\mathrm{u}}=\mathbf{Q}_{\mathbf{m}}
$$

Ideally, the solution should be coincident with the original acquired motion but, as pointed out in the literature [14], it is not, due to the unstable character of human gait and to the integration errors. Initially, a time step of $10 \mathrm{~ms}$ was adopted for the FDA, but the simulation was completely unstable. Then, the time-step size was reduced to $1 \mathrm{~ms}$. Since the IDA had been performed at $100 \mathrm{~Hz}$, additional points had to be generated, which was straightforward as B-splines had been adjusted to the adquired motion, as explained in Section 2. Using the time step of $1 \mathrm{~ms}$, the FDA was able to reproduce the motion until the $90 \%$ of the gait cycle and then drifted away.

\subsection{PD control with computed feedforward}

The second method consisted of using the same inputs as in the previous case, but including now a proportional-derivative (PD) control of the external reactions and the net joint torques so as to follow the acquired motion and avoid instabilities. The inputs provided by the controllers are in this case,

$$
\mathbf{Q}_{\mathrm{u}}=\mathbf{Q}_{\mathrm{m}}+\mathbf{K}_{\mathrm{D}}\left(\dot{\mathbf{z}}_{\mathrm{ref}}-\dot{\mathbf{z}}\right)+\mathbf{K}_{\mathrm{P}}\left(\mathbf{z}_{\mathrm{ref}}-\mathbf{z}\right)
$$


where $\mathbf{z}_{\text {ref }}$ is the vector containing the reference signals of the controllers, which are the measured values of the independent coordinates, and $\mathbf{z}$ is the vector containing the actual values of the independent coordinates. The gains of the controllers are gathered in the diagonal matrices $\mathbf{K}_{\mathrm{P}}$ and $\mathbf{K}_{\mathrm{D}}$, whose values, $k_{\mathrm{P} i}$ and $k_{\mathrm{D} i}$, associated to each independent coordinate, were adjusted by trial and error. The gain values for the different degrees of freedom of the system are shown in Table 1 as functions of two basic parameters, $k_{\mathrm{P}}$ and $k_{\mathrm{D}}$, and the masses of the corresponding body segments, $m_{j}, j=1, \ldots, \#$ of bodies.

Table 1. Selected gains for the PD controllers.

\begin{tabular}{|c|c|c|c|}
\hline \multicolumn{2}{|c|}{$k_{\mathrm{P}}=350, k_{\mathrm{D}}=1$} & $k_{\mathrm{P} i} / m_{j}$ & $k_{\mathrm{D} i} / m_{j}$ \\
\hline \multirow{3}{*}{$\begin{array}{c}\text { Translation of } \\
\text { the lumbar joint }\end{array}$} & $x$ & $k_{\mathrm{P}}$ & $k_{\mathrm{D}}$ \\
\cline { 2 - 4 } & $y$ & $6 k_{\mathrm{P}}$ & $k_{\mathrm{D}}$ \\
\cline { 2 - 4 } & $z$ & $8 k_{\mathrm{P}}$ & $8 k_{\mathrm{D}}$ \\
\hline \multicolumn{2}{|l|}{ Rotation of body $j$} & $0.009 k_{\mathrm{P}}$ & $0.003 k_{\mathrm{D}}$ \\
\hline
\end{tabular}

As it happened for the first method, if a time step of $10 \mathrm{~ms}$ was used, the simulation was completely unstable. However, using again a time step of $1 \mathrm{~ms}$, the FDA was able to reproduce the entire motion, although the results are very sensitive to the values selected for the gains of the controllers. Discrepancies between measured and calculated values for this method are in the order of $10^{-7} \mathrm{~m}$ for the translational coordinates $(x, y, z$ of the lumbar joint), $10^{-5} \mathrm{rad}$ for the angular coordinates, $10^{-2} \mathrm{~N}$ for the force components of the ground reaction, and $10^{-4} \mathrm{Nm}$ for the moment components of the ground reaction and for the net joint torques.

\subsection{Computed Torque Control}

The third method consisted of using only, as input of the FDA, the actuation provided by the so-called computed torque control (CTC) [18]. The reference signals of the controllers are the same as those already explained for the previous method. Then, the inputs provided by the controllers are,

$$
\mathbf{Q}_{\mathrm{u}}=\mathbf{M}\left[\ddot{\mathbf{z}}_{\mathrm{ref}}+\mathbf{C}_{\mathrm{D}}\left(\dot{\mathbf{z}}_{\mathrm{ref}}-\dot{\mathbf{z}}\right)+\mathbf{C}_{\mathrm{P}}\left(\mathbf{z}_{\text {ref }}-\mathbf{z}\right)\right]-\mathbf{Q}_{\mathrm{r}}
$$

where $\mathbf{C}_{\mathrm{P}}$ and $\mathbf{C}_{\mathrm{D}}$ are diagonal matrices containing the gains, $c_{\mathrm{P} i}$ and $c_{\mathrm{D} i}$, associated to each independent coordinate. As explained in [18], the error dynamics of this control method is represented by a system of second order differential equations, having $\mathbf{C}_{\mathrm{P}}$ and $\mathbf{C}_{\mathrm{D}}$ as coefficients of the proportional and first derivative terms, respectively. Therefore, imposing the relation,

$$
c_{\mathrm{D} i}=2 \sqrt{c_{\mathrm{P} i}}
$$


between the gains associated to a certain coordinate, critical damping is achieved, so that only one gain value should be tuned by coordinate. In this case, the $c_{\mathrm{P} i}$ values were adjusted.

Unlike the previous method, this one proves to be very robust with respect to the selected gains, so that the same value can be given to all the elements of $\mathbf{C}_{\mathrm{P}}$, as each controller is affected by the corresponding inertia, as shown in (8). Gain values for $\mathbf{C}_{\mathrm{P}}$ ranging between $10^{-1}$ and $10^{5}$ were tried, leading to stable simulations in all cases.

This time, the method was able to complete the simulation even with a time step of 10 ms, which represents an additional confirmation of its robustness. Although with such a time step the position errors were small, the errors in force/moment were notable. However, if the time step was set to $1 \mathrm{~ms}$ the results were excellent. For example, for a value of the $c_{\mathrm{P} i}$ elements of $10^{3}$, errors were in the order of $10^{-6} \mathrm{~m}$ for the translational coordinates ( $x, y, z$ of the lumbar joint), $10^{-4} \mathrm{rad}$ for the angular coordinates, $1 \mathrm{~N}$ for the force components of the ground reaction, and $10^{-1} \mathrm{Nm}$ for the moment components of the ground reaction and for the net joint torques. These errors are greater than those yielded by the previous method, but they can be reduced by increasing the gain values. However, some noise appears in the solution as the gains are increased.

\section{Forward dynamics: underactuated system}

As pointed out at the beginning of the previous Section, the human body does not possess actuators governing the degrees of freedom of the base body. Therefore, a new step is given towards reality in this Section, by assuming that there can be actuation in the joints, but not in the base body. From a mechanical point of view, this means that now the system is considered as underactuated. Controllers governing the actuators are to track a number of outputs, which can be trajectories, forces or a combination of both. In what follows, a CTC-like approach for underactuated systems is described [19-21], which provides the inputs of the controllers as functions of the mentioned outputs. To begin with, the equations of motion of the system (5) are reproduced here for clarity,

$$
\mathbf{M z}=\mathbf{Q}_{\mathrm{u}}+\mathbf{Q}_{\mathrm{r}}
$$

where $\mathbf{Q}_{\mathrm{u}}$ is the vector of the inputs provided by the controllers and $\mathbf{Q}_{\mathbf{r}}$ is the vector of the remaining generalized forces. Since now the controllers are less than the number of degrees of freedom of the system, equation (10) is rewritten as,

$$
\mathbf{M} \ddot{\mathbf{z}}=\mathbf{B u}+\mathbf{Q}_{\mathrm{r}}
$$

where $\mathbf{u}$ is the vector of actuations, which is projected into the space of independent coordinates through projection matrix $\mathbf{B}$.

The required outputs, $\mathbf{y}$, are considered to be either functions of the coordinates (e.g. joint trajectories), $\mathbf{y}_{1}$, or functions of the coordinates and their first derivatives (e.g. ground reactions produced by a contact model), $\mathbf{y}_{2}$, 


$$
\mathbf{y}=\left\{\begin{array}{c}
\mathbf{y}_{1}(\mathbf{z}) \\
\mathbf{y}_{2}(\mathbf{z}, \dot{\mathbf{z}})
\end{array}\right\}
$$

Differentiating (12) with respect to time (twice for $\mathbf{y}_{1}$ and once for $\mathbf{y}_{2}$ ), and substituting then $\ddot{\mathbf{z}}$ from (11) yields,

$$
\begin{gathered}
\hat{\mathbf{y}}=\left\{\begin{array}{c}
\ddot{\mathbf{y}}_{1}(\mathbf{z}) \\
\dot{\mathbf{y}}_{2}(\mathbf{z}, \dot{\mathbf{z}})
\end{array}\right\}=\left[\begin{array}{ll}
\dot{\mathbf{H}}_{1 \mathbf{z}} & \mathbf{H}_{1 \mathbf{z}} \\
\mathbf{H}_{2 \mathbf{z}} & \mathbf{H}_{2 \dot{z}}
\end{array}\right]\left\{\begin{array}{l}
\dot{\mathbf{z}} \\
\ddot{\mathbf{z}}
\end{array}\right\}=\left[\begin{array}{l}
\dot{\mathbf{H}}_{1 \mathbf{z}} \\
\mathbf{H}_{2 \mathbf{z}}
\end{array}\right] \dot{\mathbf{z}}+\left[\begin{array}{l}
\mathbf{H}_{1 \mathbf{z}} \\
\mathbf{H}_{2 \dot{\mathbf{z}}}
\end{array}\right] \ddot{\mathbf{z}}= \\
=\mathbf{A} \dot{\mathbf{z}}+\mathbf{D} \ddot{\mathbf{z}}=\mathbf{A} \dot{\mathbf{z}}+\mathbf{D} \mathbf{M}^{-1}\left(\mathbf{B u}+\mathbf{Q}_{\mathrm{r}}\right)
\end{gathered}
$$

so that the vector of actuations $\mathbf{u}$ can be worked out from Eq. (13) as,

$$
\mathbf{u}=\left(\mathbf{D} \mathbf{M}^{-1} \mathbf{B}\right)^{-1}\left(\hat{\mathbf{y}}-\mathbf{A} \dot{\mathbf{z}}-\mathbf{D} \mathbf{M}^{-1} \mathbf{Q}_{\mathrm{r}}\right)
$$

Now, calling $\mathbf{P}=\mathbf{D} \mathbf{M}^{-1} \mathbf{B}$, and considering that feedback is introduced for the outputs, it results,

$$
\mathbf{u}=\mathbf{P}^{-1}\left(\left\{\begin{array}{c}
\ddot{\mathbf{y}}_{1}^{\text {ref }}+\mathbf{C}_{\mathrm{D}}\left(\dot{\mathbf{y}}_{1}^{\mathrm{ref}}-\dot{\mathbf{y}}_{1}\right)+\mathbf{C}_{\mathrm{P}}\left(\mathbf{y}_{1}^{\mathrm{ref}}-\mathbf{y}_{1}\right) \\
\dot{\mathbf{y}}_{2}^{\mathrm{ref}}+\mathbf{K}_{\mathrm{P}}\left(\mathbf{y}_{2}^{\mathrm{ref}}-\mathbf{y}_{2}\right)
\end{array}\right\}-\mathbf{A} \dot{\mathbf{z}}-\mathbf{D} \mathbf{M}^{-1} \mathbf{Q}_{\mathrm{r}}\right)
$$

where super-index ref indicates the desired values of the outputs, different from the current ones (without super-index), and $\mathbf{C}_{\mathrm{D}}, \mathbf{C}_{\mathrm{P}}$ and $\mathbf{K}_{\mathrm{P}}$ are diagonal matrices containing the gains associated to each output.

If the number of outputs is equal to that of actuators, matrix $\mathbf{P}$ is square and the required inputs can be determined from (15). If the number of outputs is greater than that of actuators, the required outputs can be satisfied in a minimum squares sense only, the system of equations to be solved being,

$$
\mathbf{u}=\left(\mathbf{P}^{\mathrm{T}} \mathbf{W P}\right)^{-1} \mathbf{P}^{\mathrm{T}} \mathbf{W}\left(\left\{\begin{array}{c}
\ddot{\mathbf{y}}_{1}^{\mathrm{ref}}+\mathbf{C}_{\mathrm{D}}\left(\dot{\mathbf{y}}_{1}^{\mathrm{ref}}-\dot{\mathbf{y}}_{1}\right)+\mathbf{C}_{\mathrm{P}}\left(\mathbf{y}_{1}^{\mathrm{ref}}-\mathbf{y}_{1}\right) \\
\dot{\mathbf{y}}_{2}^{\mathrm{ref}}+\mathbf{K}_{\mathrm{P}}\left(\mathbf{y}_{2}^{\text {ref }}-\mathbf{y}_{2}\right)
\end{array}\right\}-\mathbf{A} \dot{\mathbf{z}}-\mathbf{D} \mathbf{M}^{-1} \mathbf{Q}_{\mathrm{r}}\right)
$$

with $\mathbf{W}$ the weight diagonal matrix which assigns more weight to more relevant outputs.

\subsection{External reactions from inverse-dynamics analysis}

Since actuation can be provided at joints only, the external reactions should be supplied by a foot-ground contact model that evaluates them during the simulation as functions of feet position and velocity. However, this approach is highly challenging, since the discrepancies between the reactions yielded by the contact model and the true ones can be substantial, thus compromising control stability. Therefore, to start addressing the problem in a more feasible way, the external reactions obtained from IDA (Section 4) were introduced to the feet. The 3D model was considered in this case. The inputs were 
the actuations at the 51 angular degrees of freedom associated to the joints, and the outputs were the corresponding angular coordinates, i.e. inputs and outputs affect to the same model coordinates. The gain values in matrix $\mathbf{C}_{\mathrm{P}}$ of (15) were all set to $10^{3}$, while the values in matrix $\mathbf{C}_{D}$ were obtained by imposing relation (9), yielding a value of 63.24. Given that the outputs were coordinates, only the upper part of (15) was required.

With a time step of $10 \mathrm{~ms}$, the simulation failed. However, using a time step of $1 \mathrm{~ms}$, the simulation worked well. RMS errors were of $6.09 \mathrm{e}-4 \mathrm{~m}$ for the translational coordinates ( $x, y, z$ of the lumbar joint), 8.87e-4 rad for the angular coordinates, and $1.5 \mathrm{e}-3 \mathrm{Nm}$ for the net joint torques (errors in the external reactions do not apply to this case as they were imposed).

\subsection{External reactions from inverse-dynamics analysis with perturbation}

In order to go one step further and take into account that discrepancies are to be introduced when a contact model is used, a perturbation was added to the external reactions obtained from IDA. The perturbation consisted of a constant force of $1 \mathrm{~N}$ applied on the lumbar joint of the 3D model in the positive longitudinal direction ( $x$-coordinate), which approximately represents $1 \%$ of the total longitudinal force.

Initially, the inputs and outputs adopted were the same as in the previous sub-section, as were the values of the gains. The simulation could be run with a time step of $1 \mathrm{~ms}$, leading to RMS errors below 1e-3 rad in the outputs. Since the motion of the base body was not considered as output, the perturbation had the effect of accelerating the longitudinal pelvis motion with respect to the acquired motion. In Fig. 3 left, it can be seen that the error in this magnitude grows with time, as this coordinate was not a control output, and the perturbed model overtakes its unperturbed counterpart. However, the error in, for example, the flexion angle between pelvis and trunk was kept very low, as a consequence of being a control output.
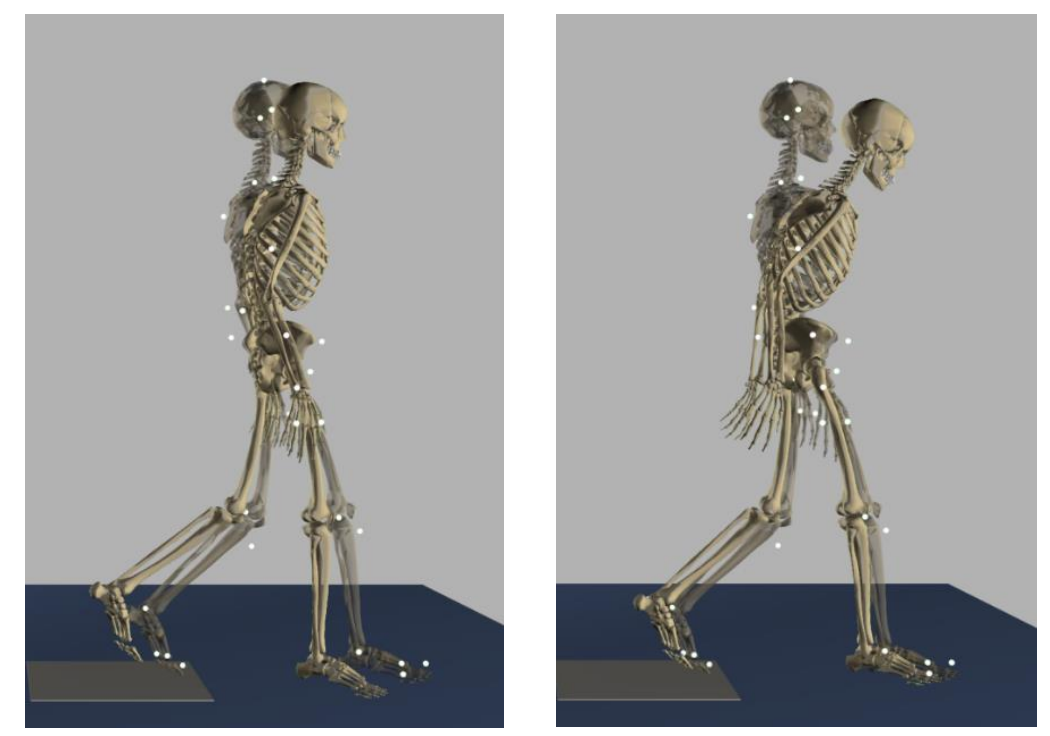

Figure 3. Postures of the model at the end of the simulation without control of the longitudinal coordinate of the lumbar joint (left) and in the case of substituting, in the list of control outputs, the flexion angle between pelvis and trunk by the longitudinal 
coordinate of the lumbar joint (right). In both pictures, the two superimposed images correspond to the same time point.

Secondly, the $x$-coordinate of the lumbar joint was included in the list of control outputs but, in order to keep as many outputs as inputs, the flexion angle between pelvis and trunk was removed from the list. Again, the simulation could be completed for a time step of 1 $\mathrm{ms}$, yielding RMS errors below 1e-3 $\mathrm{m}$ or rad in the outputs at configuration level. This means that the longitudinal motion of the lumbar joint was correctly reproduced, since it had been included as a control output. However, the flexion angle between pelvis and trunk experimented a drift, as a result of not having been considered as a control output. These behaviors are represented in Fig. 3 right.

\subsection{External reactions from contact model}

In this sub-section, a contact model was considered at the interface between foot and ground to generate the external reactions. Two options are typically available for the contact model: a force model or a constraint model. If a force model is chosen, the system is certainly underactuated, and control methods for such types of systems must be used, as the one described at the beginning of this Section, facing the problem of the unstable nature of gait. If a constraint model is selected seeking to have a fully-actuated system at all times, constraints must be alternatively imposed to the feet (thus perturbing the continuous motion they experience during gait, even at the stance phase), and the impact at landing must be dealt with in some way. Therefore, a force model was applied in this work because it seems to be more consistent with reality.

Moreover, there is a problem that must be faced when using foot-ground force contact models in the FDA of acquired gait motions: the selection of the contact model parameters and, more importantly, of the feet boundaries. A not sufficiently good location of feet boundaries can yield huge contact forces that make the simulation fail. Therefore, an optimization method to select the mentioned characteristics of the contact model, similar to the one proposed in [22], is required to be applied as a pre-processing stage, prior to the FDA, to ensure reasonable contact forces during the simulation.

Given the challenging character of the objective pursued in this sub-section, it was firstly addressed for the 2D model, for which several choices of outputs and their corresponding weights were evaluated. Then, the 3D case was addressed.

\subsubsection{D human model}

The planar model described in sub-section 2.2 is used in this sub-section. It is reminded here that the configuration vector $\mathbf{z}$ of 14 independent coordinates that was selected for this model is formed by the two Cartesian coordinates of the hip and the angle between vertical axis and trunk (three degrees of freedom of the base body), along with the 11 relative angles illustrated in Fig. 2 right,

$$
\mathbf{z}^{\mathrm{T}}=\left\{\begin{array}{llllllllllllll}
x_{4} & y_{4} & \alpha_{0} & \alpha_{1} & \alpha_{2} & \alpha_{3} & \alpha_{4} & \alpha_{5} & \alpha_{6} & \alpha_{7} & \alpha_{8} & \alpha_{9} & \alpha_{10} & \alpha_{11}
\end{array}\right\}
$$

Regarding the foot-ground force contact model, the nonlinear volumetric contact model proposed in [23] was used, for which the normal and tangential contact forces are defined as, 
where $V$ is the interpenetration volume, $k_{\mathrm{h}}$ is the hyper-volumetric pseudo-stiffness, $h$ is an exponent which depends on the volumetric stiffness and geometrical properties, $a_{\mathrm{h}}$ is the foundation stiffness multiplied by the damping, $v_{\mathrm{cn}}$ and $v_{\mathrm{ct}}$ are the normal and tangential velocities at the centroid of the deformed volume, respectively, $\mu$ is the friction coefficient, $\mu_{\mathrm{f}}$ is the asymptotic friction coefficient, and $v_{\mathrm{s}}$ is a shape factor. Reasonable values of parameters $h, \mu_{\mathrm{f}}$, and $v_{\mathrm{s}}$ where taken from [23], whereas values for $k_{\mathrm{h}}$ and $a_{\mathrm{h}}$ were obtained by trial and error, bearing in mind that interpenetration areas are considered in the 2D case instead of volumes. The values of all the parameters are listed in Table 2.

Table 2. Parameters of the foot-ground contact model.

\begin{tabular}{|c|c|c|c|c|}
\hline$k_{\mathrm{h}}$ & $h$ & $a_{\mathrm{h}}$ & $\mu_{\mathrm{f}}$ & $v_{\mathrm{s}}$ \\
\hline $10^{5}$ & 0.79 & $2 \cdot 10^{6}$ & 0.34 & 0.034 \\
\hline
\end{tabular}

The optimization pre-process to set the feet boundaries is as follows. For each foot, a local reference system $(\bar{x}, \bar{y})$ was defined as shown in Fig. 4 , with the origin at the ankle and the $\bar{x}$-axis horizontal in the support position. Then, 10 equally-spaced points spanning the whole foot were taken along the $\bar{x}$-axis. The $\bar{y}$-coordinates of these points, $\bar{y}_{i}, \quad i=1,2, \ldots, 10$, which served to define the foot boundary through cubic splines, were the design variables of the optimization problem. The cost function to be minimized was the discrepancy between the histories of the ground reactions provided by the foot-ground contact model and those obtained from the IDA, when imposing to the foot the same recorded motion. Normal and tangential forces, as well as the reaction moment, were considered in the cost function, scaling the reaction moment by a factor of 100 in order to balance the weight of the three components. The ga genetic algorithm from Matlab [24] was used, for which no initial guess is required, and the resulting feet boundaries are depicted in Fig. 5.

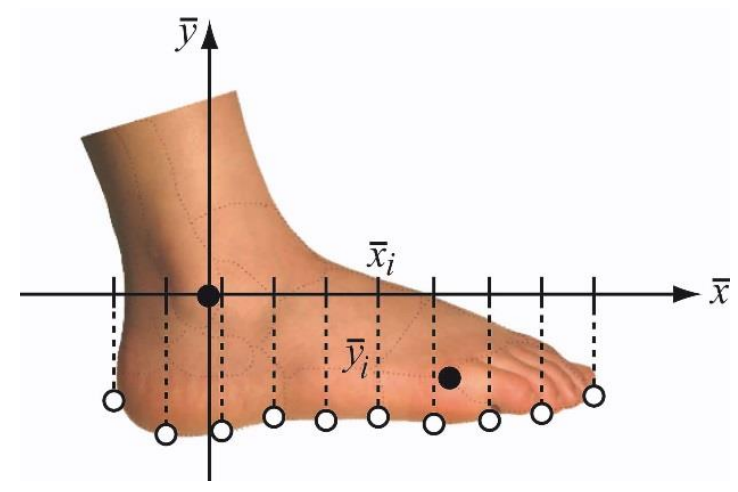

Figure 4. Foot boundary definition in the $2 \mathrm{D}$ case. 


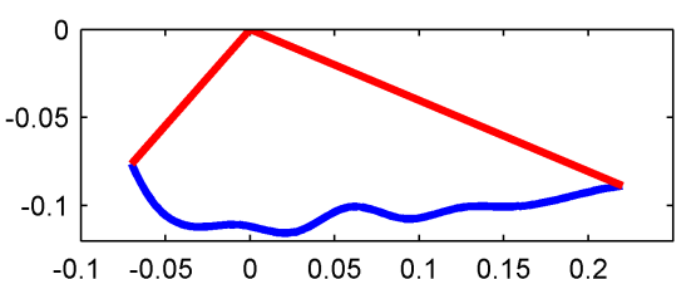

Figure 5. Feet boundaries obtained from the optimization pre-process.

Once the model was completely defined, the formulations for underactuated systems described at the beginning of this Section were applied.

Several alternatives in the choice of the outputs were investigated, looking for the one that yielded the best agreement between the result of the forward-dynamics simulation and the acquired motion. Here, the most representative options are described. The gain values in matrix $\mathbf{C}_{\mathrm{P}}$ of (15) and (16) were all set to $10^{3}$, while those in matrix $\mathbf{C}_{\mathrm{D}}$ were obtained by imposing relation (9), thus resulting in a value of 63.25 ; the values in matrix $\mathbf{K}_{\mathrm{P}}$ were all set to $10^{3}$. In all cases, the simulations were run with a constant time step of $1 \mathrm{~ms}$. Remember that the human model considered had 14 degrees of freedom and 11 inputs (joint actuators).

The first strategy tested (case 1) was to define as many outputs as inputs, i.e. 11, choosing as outputs some 11 coordinates from the configuration vector $\mathbf{z}$ defined in (17). The selected outputs were the joint relative angles, while the three coordinates of the base body (trunk) were left free. The upper part of (15) was used to calculate the required inputs along the simulation.

The second strategy tested (case 2) was to define more outputs than inputs, choosing as outputs the 14 coordinates of the configuration vector $\mathbf{z}$ defined in (17). The upper part of (16) was used to calculate the required inputs along the simulation. In this case, the weights for the outputs had to be decided, and used to build the weight matrix $\mathbf{W}$. An equal weight value of 1 was chosen for all the outputs (other distributions were tested, but no significant differences were observed).

The third strategy tested (case 3) was to define more outputs than inputs, as in case 2, but choosing as outputs the 14 coordinates of the configuration vector $\mathrm{z}$ defined in (17) plus the three ground reaction components (normal and tangential forces, and reaction moment) at each foot, i.e. 6 ground reaction components, leading to a total number of 20 outputs [21]. The whole equation (16) was used to calculate the required inputs along the simulation. In this case, the weights required to build matrix $\mathbf{W}$ had to be decided too: the kinematic outputs were assigned a weight value 1, the ground normal reaction forces were assigned a weight value $10^{-3}$, and both the ground tangential reaction forces and the reaction moments were assigned a weight value of $10^{-2}$, so that the 20 outputs had a similar order of magnitude (as in case 2, other distributions were tested without relevant changes observed).

The RMS errors between the acquired motion (reference) and the result of the forwarddynamics simulation (cases 1, 2 or 3) are presented in Table 3. 
Table 3. RMSE of the forward-dynamics simulation with respect to the acquired motion.

\begin{tabular}{|c|c|c|c|}
\hline RMSE & Case 1 & Case 2 & Case 3 \\
\hline Translational coordinates $(\mathrm{m})$ & $1 \mathrm{e}-2$ & $9.7 \mathrm{e}-3$ & $7.79 \mathrm{e}-2$ \\
\hline Angular coordinates $(\mathrm{rad})$ & $3.2 \mathrm{e}-3$ & $2.7 \mathrm{e}-3$ & 0.6 \\
\hline Reaction forces $(\mathrm{N})$ & 163.99 & 164.66 & 139.36 \\
\hline Reaction moments $(\mathrm{Nm})$ & 32.83 & 32.97 & 38.55 \\
\hline Net joint torques $(\mathrm{Nm})$ & 27.09 & 27.14 & 288.82 \\
\hline
\end{tabular}

More detailed results are presented in the following. Figure 6 shows the histories of the three coordinates of the base body (trunk) and the right hip angle, for the three cases studied, and compares them with the result of the IDA, taken as reference.
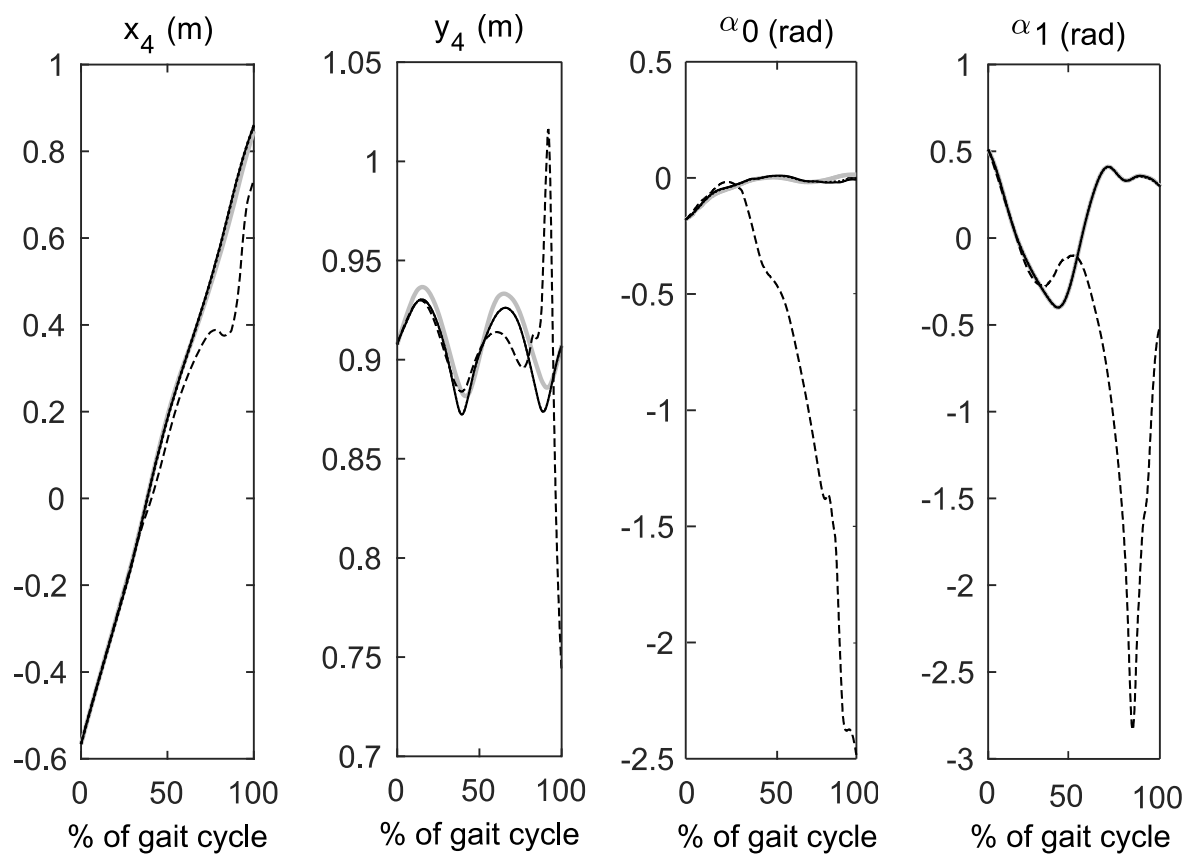

Figure 6. Coordinates of the base body (trunk) and right hip angle, obtained with different control strategies (case 1, solid; case 2, dotted; case 3, dashed) vs reference (IDA, grey solid). Plots of cases 1 and 2 are almost coincident.

Figure 7 gathers the histories of the normal ground reaction force at the left foot for the three cases studied, and compares them with the result of the IDA, taken as reference. Note that the simulation started with the toe-off of the left foot. 


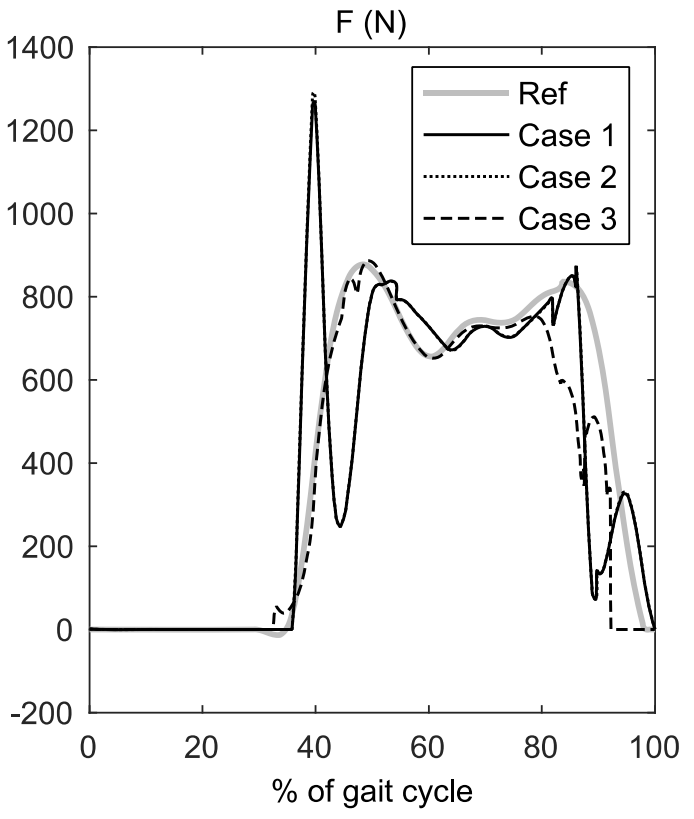

Figure 7. Normal ground reaction force obtained with different control strategies vs reference (IDA). Plots of cases 1 and 2 are almost coincident.

In the last two figures, it can be seen that cases 1 and 2 provide good motion correlation, although some peaks can be observed in the ground reactions. Conversely, case 3 yields an excellent correlation of the ground reactions, at the prize of being far from following the motion and making the model fall. Therefore, it was found that the best motion results were obtained when the ground reactions were not considered as outputs.

To provide a clearer illustration of the obtained gaits, Fig. 8 compares the resulting model motion for case 2 with the acquired motion.

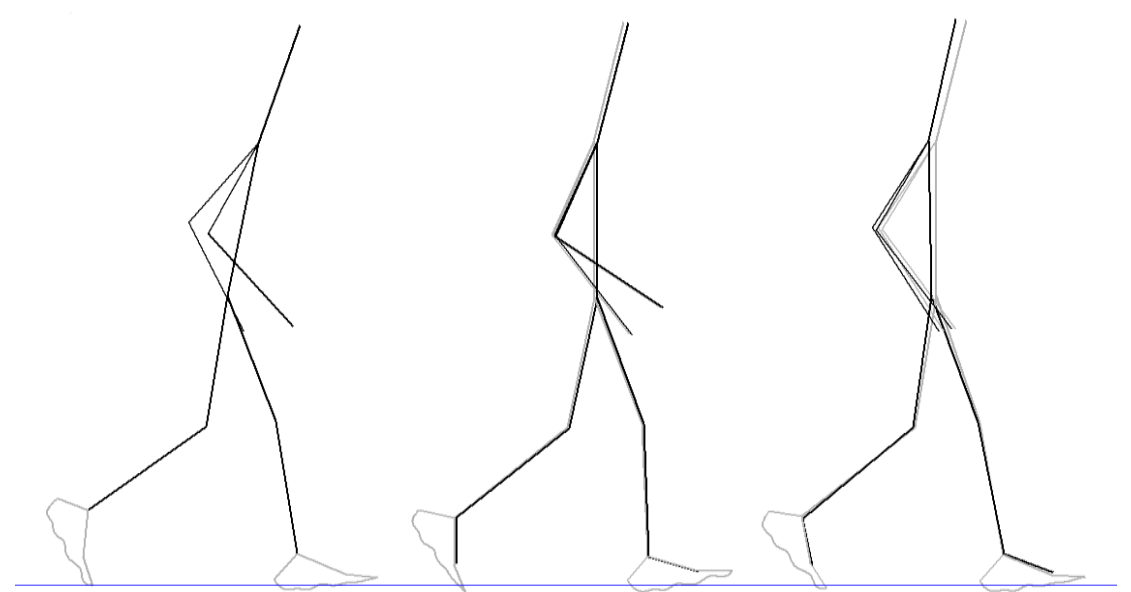

Figure 8. Model motion in case 2 (black) vs reference (IDA, grey).

Although, looking at Fig. 8, it could be thought that discrepancy between the acquired and simulated motion could have been caused by the existence of some sliding between foot and ground due to the adopted contact force model, implementation of a stick-slip model led to similar results. 


\subsubsection{D human model}

The three-dimensional human model described in sub-section 2.1 was used in this subsection. It is reminded here that the configuration vector $\mathbf{z}$ of 57 independent coordinates that was selected for this model was formed by the three Cartesian coordinates of the lumbar joint plus the 18 sets of three angles defining the orientation of the bodies (Fig. $1)$.

The foot-ground force contact model was the same described for de $2 \mathrm{D}$ case, with the difference that in this case the feet boundaries were 3D spline surfaces.

The optimization pre-process to set the feet boundaries was also analogous to that of the $2 \mathrm{D}$ case. Splines were generated from a grid of $7 \times 3$ points, whose local $\bar{z}$-coordinates were optimized. However, in the 3D case it was very difficult to obtain feet boundaries which led to a stable behavior of the model in forward dynamics: small differences in the feet boundaries caused drifts in moments or foot placement that made the model fall. Therefore, some tiny hand-made adjustments to the optimization results were necessary to finally achieve a stable behavior in the forward-dynamics simulation. Figure 9 shows the shapes of the 3D spline boundaries obtained, and the points of the grid.
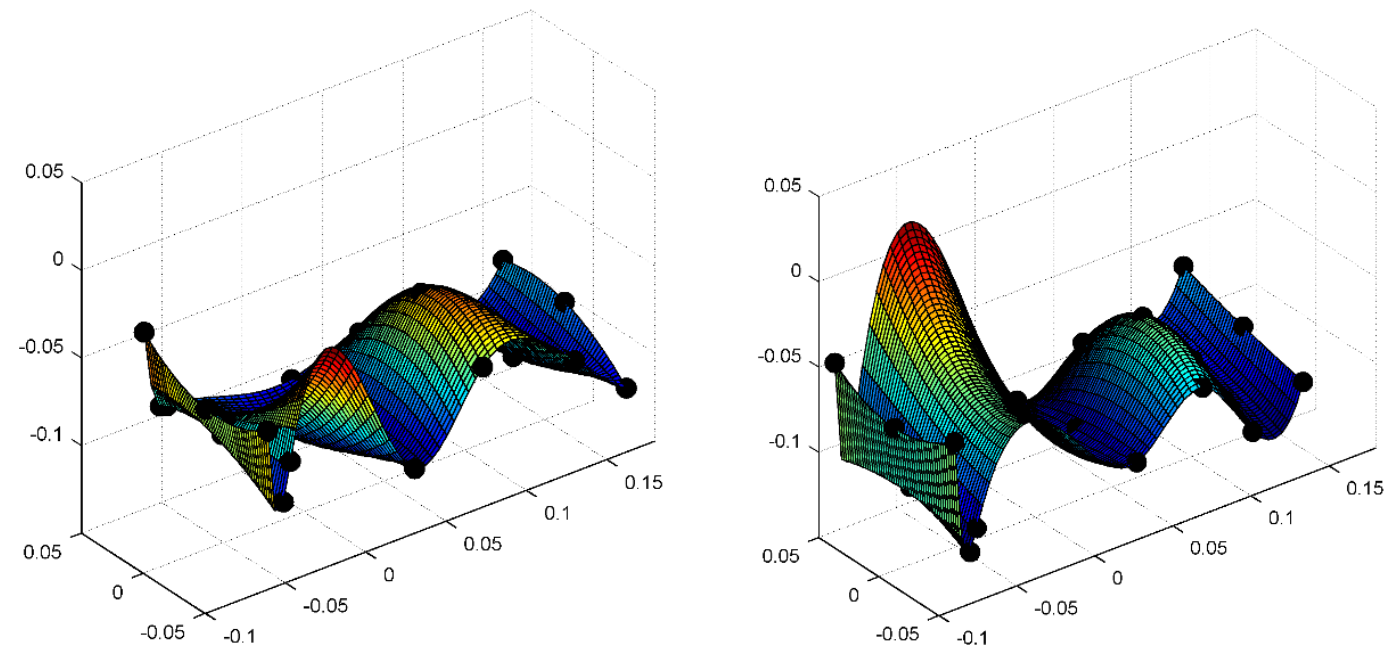

Figure 9. Feet boundaries with 3D splines obtained from optimization for the right foot (right) and left foot (left).

In this case, the strategy adopted was the one which provided the best motion tracking in the planar case, i.e. to define more outputs than inputs choosing as outputs the 57 coordinates of the configuration vector $\mathbf{z}$. The upper part of (16) was used to calculate the required inputs along the simulation, assigning to the gains in matrix $\mathbf{C}_{\mathrm{P}}$ a value of $10^{3}$ , and obtaining the gains in matrix $\mathbf{C}_{\mathrm{D}}$ by application of (9), yielding a value of 63.25. All the weights needed to build matrix $\mathbf{W}$ were set to 1 . The time step used to run the simulation was $1 \mathrm{~ms}$. 

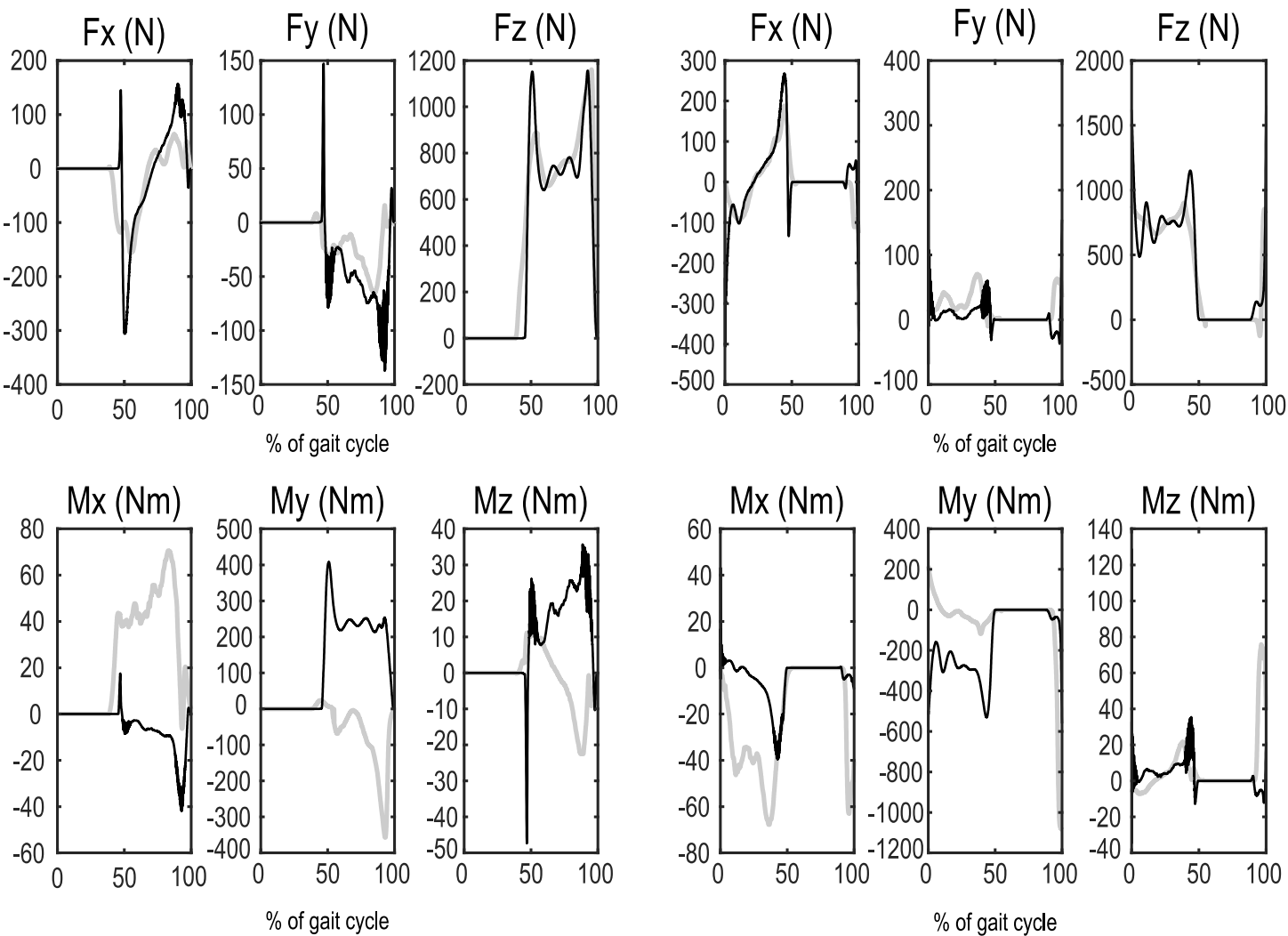

Figure 10. Comparison of contact reactions (forces and moments) obtained from forward-dynamics simulation (black) and from IDA (grey) for the right foot (right) and left foot (left).

As can be seen in Fig. 10, big discrepancies were found between the ground reactions generated when the model is run in forward dynamics and those obtained from the IDA. Despite this, the controllers managed to follow the acquired motion without falling during an entire gait cycle, with RMS errors of $0.0476 \mathrm{~m}$ for the translational coordinates $(x, y$, $z$ of the lumbar joint), 0.0117 rad for the angular coordinates, $110.84 \mathrm{~N}$ for the force components of the resultant external reaction, $14.08 \mathrm{Nm}$ for the moment components of the resultant external reaction and $14.66 \mathrm{Nm}$ for the internal net joint torques. It must be noted that discrepancies grow with time, being greater for the left foot, which is second in touching down. 

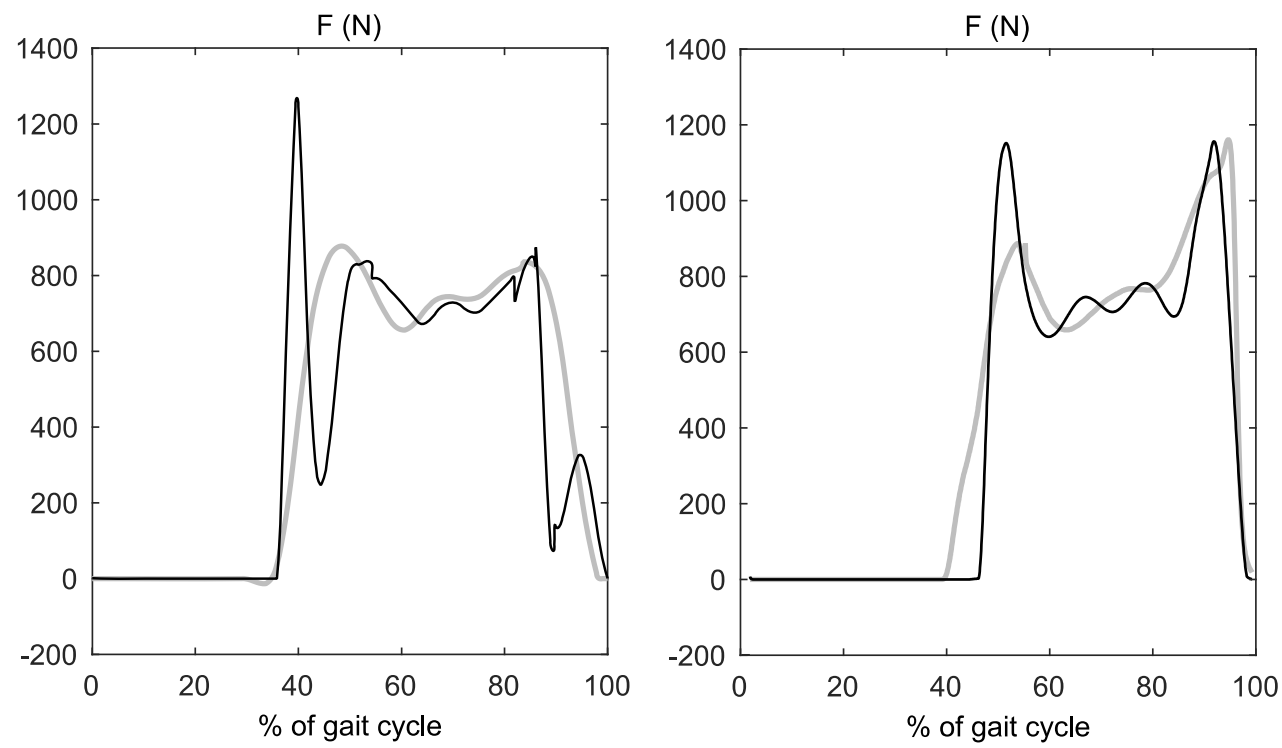

Figure 11 . History of the left-foot normal contact force provided by forward-dynamics simulation (black) and IDA (grey) for the 2D (left) and 3D (right) models.

In Figure 11, the history of the normal contact force for the left foot provided by Figure 7 for the planar model (case 2) is compared with the history of the same foot provided by Figure 10 left for the 3D model. Since the experiment is the same, the comparison gives an idea of how the different modeling affects to the results.
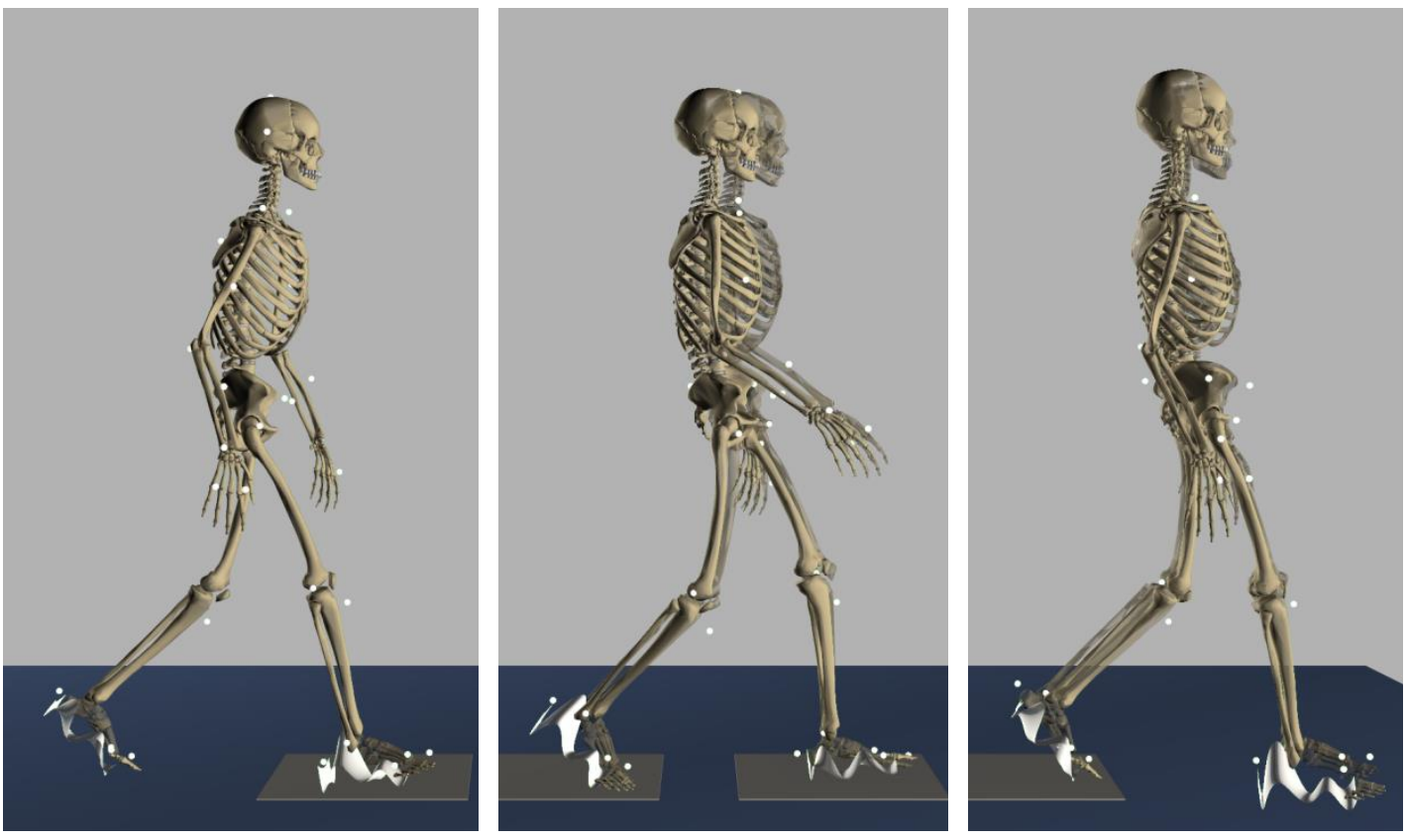

Figure 12. Postures of the model at the beginning (left), half (center) and end (right) of the forward-dynamics simulation compared with those of the acquired motion. In the three pictures, the two superimposed images correspond to the same time point. The feet boundaries considered are also illustrated.

Figure 12 shows the comparison between the motion obtained by forward-dynamics simulation and the acquired motion. 


\section{Application to assisted gait}

The previously described approaches were also applied to the forward-dynamics analysis of the gait of a spinal cord injured subject assisted by orthoses and crutches. The subject was an adult female 41 years old, mass $65 \mathrm{~kg}$ and height $1.52 \mathrm{~m}$ with injury at thoracic vertebra 11 (T11). In the experiment, she was wearing a pair of passive knee-ankle-foot orthoses while walking over two embedded force plates (AMTI, AccuGait sampling at $100 \mathrm{~Hz}$ ) with the help of two instrumented crutches. Her motion was captured by 12 optical infrared cameras (Natural Point, OptiTrack FLEX:V100 also sampling at $100 \mathrm{~Hz}$ ) that computed the position of 43 optical markers, as illustrated in Fig. 13 (left). The study was approved by the institutional ethical committee and the subject gave her informed consent.
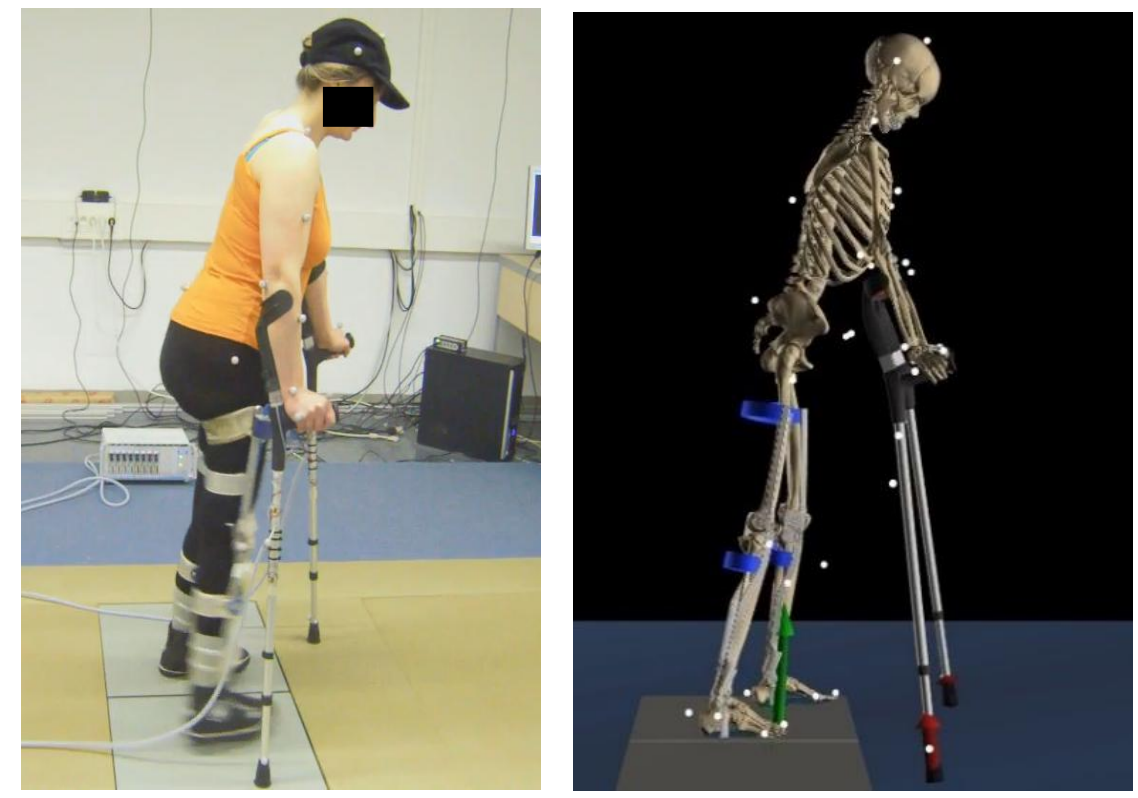

Figure 13. Gait of spinal cord injured subject assisted by passive orthoses and crutches: acquired motion and computational model.

The orthoses were a pair of standard leg braces that allow knee locking during gait so as to avoid knee flexion under the subject's weight in the support phase, and feature polymeric parts at ankle-foot level that provide ankle stiffness to prevent foot drop in the swing phase. One of them is shown in Fig. 14. 
Instrumented crutches were used in order to measure the ground reactions. For this purpose, extensometry was the selected technique by means of strain gauge-based load cells. Assuming that only a force reaction exists at the tip center, four Wheatstone bridges were required at each crutch to measure the three components of the force at the tip and the cuff reaction on the subject's forearm. Additionally, three reflective markers were placed on each crutch to provide its position for the optical motion capture. The location of load cells and markers is illustrated in Fig. 15. More details about the instrumentation of the crutches can be found in [15].

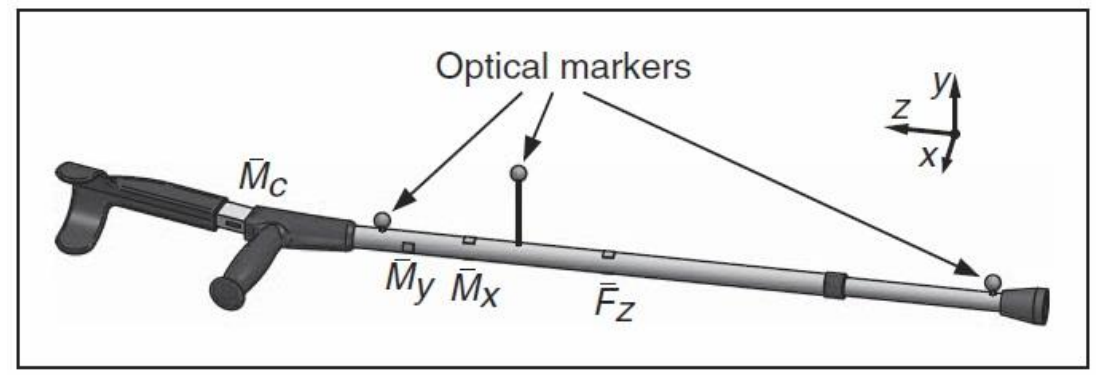

Figure 15. Instrumented crutch.

The computational model used was the same three-dimensional model described in subsection 2.1 to which some additions were made in order to include orthoses and crutches.

The crutches were introduced in the model as rigid bodies in natural coordinates. They were modeled using two points, one located at the intersection between the crutch bar and the handle (shared with the hand and, hence, already in the model), and another one located at the center of mass of the crutch, and two orthogonal unit vectors, one pointing forward and another one orthogonal to the first unit vector and to the crutch axis, thus adding a total of 18 variables to the model (two points and four unit vectors). The 
inclusion of the crutches did not add any degree of freedom to the system, since they were considered as clamped to the hands.

Regarding the orthoses, two options were considered, depending on whether the orthoses were fused with the models of the subject's legs or modeled as independent bodies.

\subsection{Orthosis model embedded in lower limb model}

In the first option, the orthoses were taken into account in the model by altering the inertia properties of the thighs, calves and feet accordingly, and by including torsional springdamper elements at knee and ankle levels to represent the locking and anti-foot-drop mechanisms, respectively.

Therefore, the resulting 3D human model including orthoses (as embedded links) and crutches possessed 57 degrees of freedom and was defined by a total of 246 mixed (natural and angular) dependent coordinates. As in the previous cases where the 3D human model was used, the configuration vector $\mathbf{z}$ of 57 independent coordinates was formed by the three Cartesian coordinates of the lumbar joint plus the 18 sets of three angles defining the orientation of the bodies (Fig. 1).

\subsubsection{Fully actuated system}

The first approach to carry out the FDA of the acquired gait motion consisted of using trajectory tracking controllers associated to all the system degrees of freedom and, more specifically, the CTC algorithm explained in sub-section 5.3.

Eq. (8) was used to calculate the required inputs along the simulation, assigning to the gains in matrix $\mathbf{C}_{P}$ a value of $10^{3}$ and obtaining the gains in matrix $\mathbf{C}_{D}$ by application of (9), yielding a value of 63.25. The simulation was run with a constant time step of 1 ms.

The obtained results showed the same accuracy levels as those obtained for the healthy subject in sub-section 5.3.

Figure 16 gathers the torques at knee and ankle levels exerted by the controller and the passive elements, and compares their addition with the total torques obtained from IDA: plots of both magnitudes cannot be distinguished in the graphs due to their good agreement. 

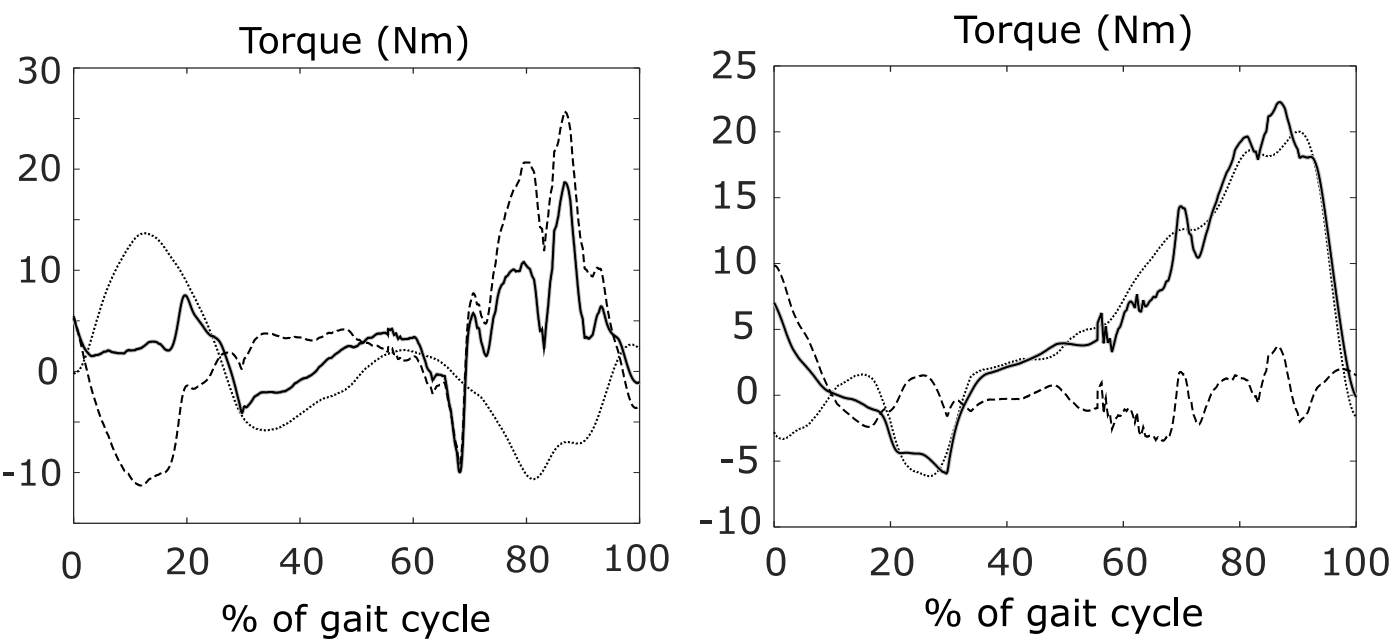

Figure 16. Torques in the right leg: knee level (left), ankle level (right). Torque exerted by orthosis (dotted), torque exerted by controller (dashed), total torque (addition of the former two, black solid), torque obtained from IDA (reference, grey solid). The black solid and grey solid plots are coincident.

\subsubsection{Underactuated system}

The alternative approach to carry out the FDA of the acquired gait motion is to use controllers acting at the joints only, and not in the base body, thus considering the system as underactuated, as explained in Section 6. This approach implies defining contact models for the system bodies interacting with the environment, i.e. the subject's feet and the crutches.

Given that this assisted gait is more stable than the healthy one, due to the stabilizing effect of the crutches, a simpler foot-ground contact model could be applied. A set of four spheres (one attached to the forefoot and three to the hindfoot) featuring the HuntCrossley normal contact force model and the bristle tangential force model proposed in [25] was used. The parameters of the contact model (for each sphere: local coordinates of the center, radius and contact parameters), were found by optimization using the $g a$ genetic algorithm from Matlab according to the approach proposed in [22].

Regarding the crutch-ground contact, it was modeled as a sphere at the tip of the crutch to which the normal contact force presented in [26] and the bristle tangential contact force proposed in [25] were applied. The contact model parameters were found by optimization using the $g a$ genetic algorithm from Matlab as described in [22].

In this case, the strategy adopted was the same applied in the 3D gait of a healthy subject in sub-section 6.3.2, i.e. to define more outputs than inputs, choosing as outputs the 57 coordinates of the configuration vector $\mathbf{z}$. Therefore, the upper part of (16) was used to calculate the required inputs along the simulation, assigning to the gains in matrix $\mathbf{C}_{\mathrm{P}}$ a value of $10^{3}$ and obtaining the gains in matrix $\mathbf{C}_{\mathrm{D}}$ by application of (9), yielding a value of 63.25. The weights for the outputs needed to build weight matrix $\mathbf{W}$ were all set to 1 . The time step used to run the simulation was $1 \mathrm{~ms}$. 

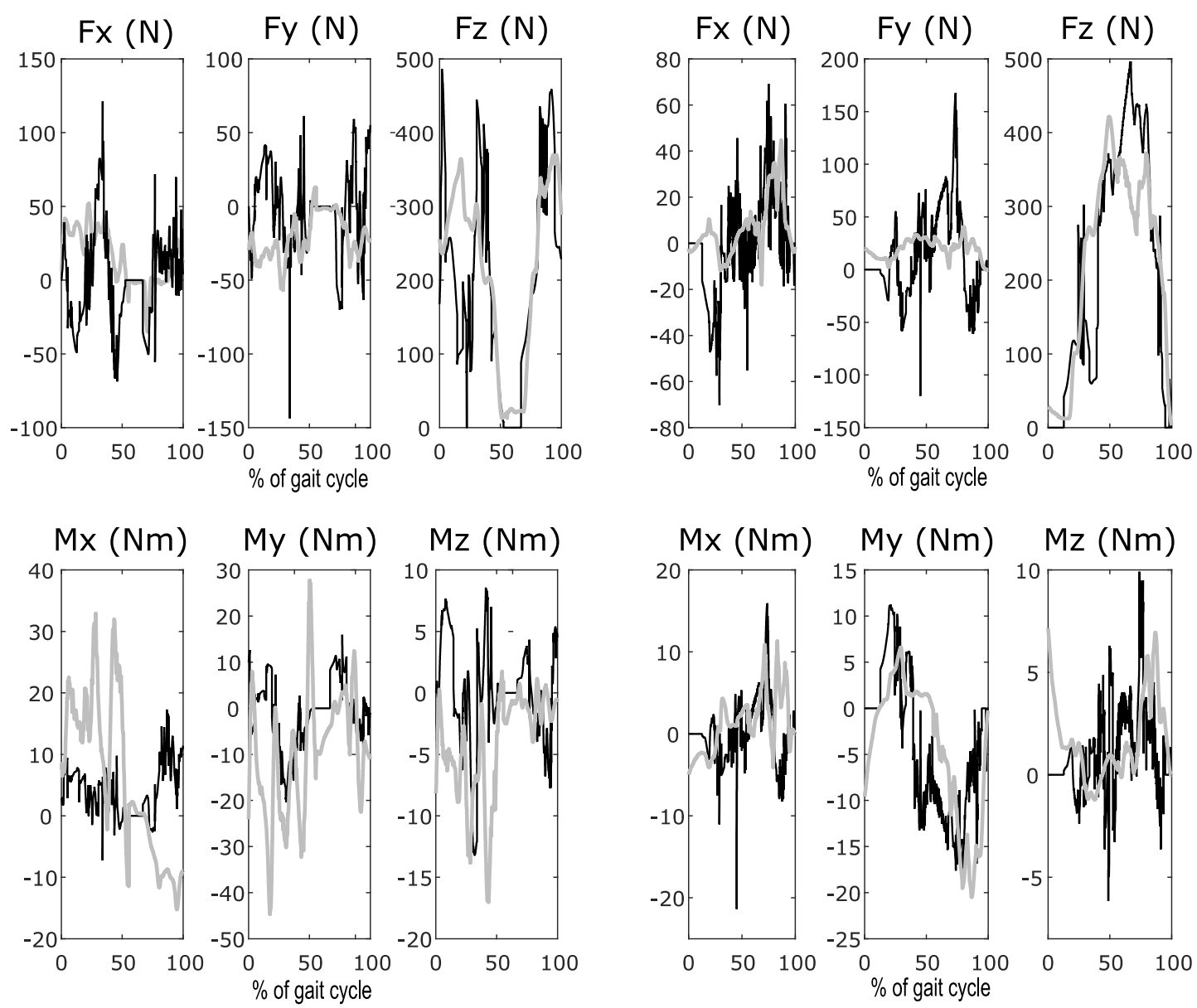

Figure 17. Comparison of ground reactions (forces and moments) obtained from forward-dynamics simulation (black) and from IDA (grey) for the right foot (right) and left foot (left).

Unlike what happened with the healthy subject, the more stable nature of the gait of the injured subject provided by the additional contacts at the crutches, made it possible that the foot-ground and crutch-ground contact models obtained through optimization were directly usable in forward dynamics, yielding a good trajectory tracking without the necessity of any hand-made tuning. As can be seen in Fig. 17, notable discrepancies were found between the ground reactions generated when the model was run in forward dynamics and those obtained from the IDA. Despite this, the controllers managed to follow the acquired motion without falling, as illustrated in Fig. 18, with RMS errors of $0.017 \mathrm{~m}$ for the translational coordinates ( $x, y, z$ of the lumbar joint $), 4.1 \cdot 10^{-3} \mathrm{rad}$ for the angular coordinates, $46.03 \mathrm{~N}$ for the force components of the external reaction, $11.08 \mathrm{Nm}$ for the moment components of the external reaction and $16.57 \mathrm{Nm}$ for the net joint torques. 

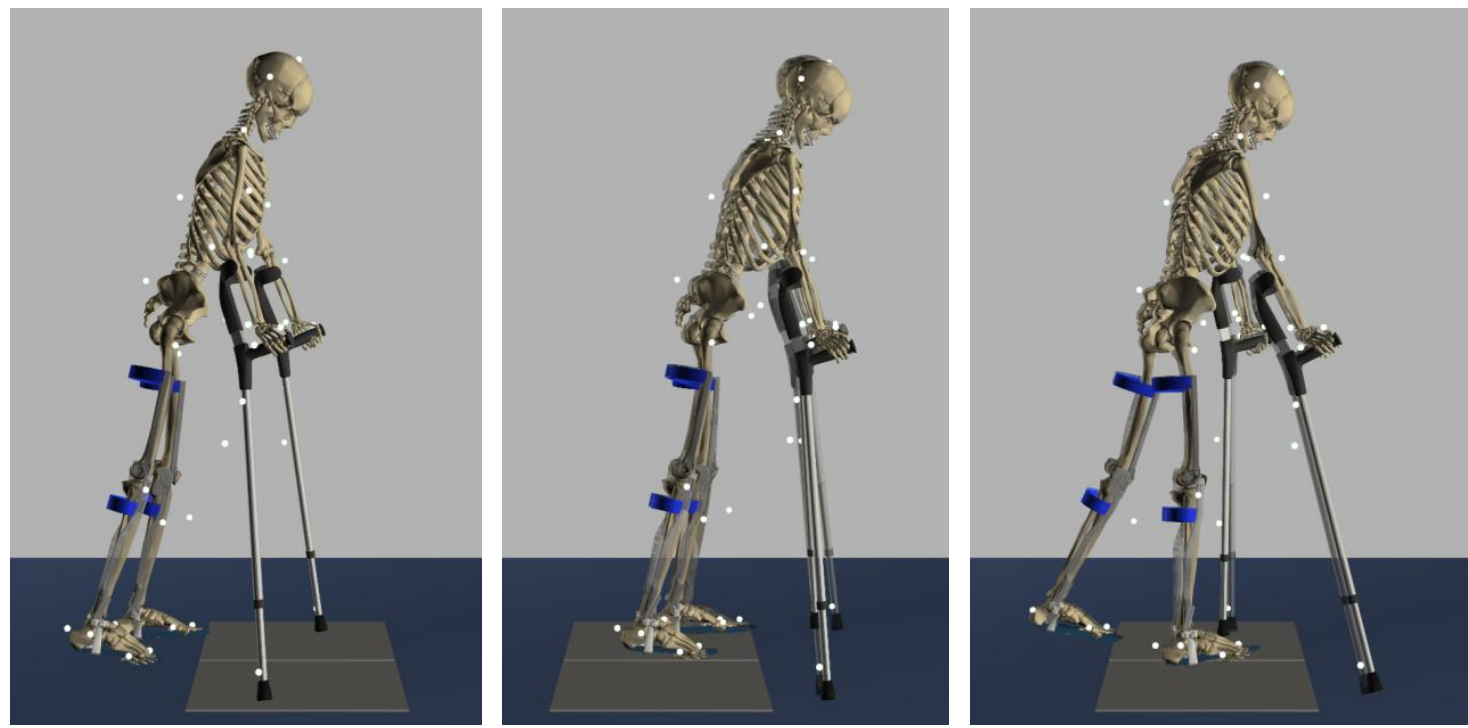

Figure 18. Postures of the model at the beginning (left), half (center) and end (right) of the forward dynamic simulation compared with those of the acquired motion. In the three pictures, the two superimposed images correspond to the same time point.

Figure 19 shows the torques at knee and ankle levels exerted by the controller and the passive elements, and compares their addition with the total torques obtained from IDA. Unlike what happened in sub-section 7.1.1, where full actuation was applied, this time the total torques present significant discrepancies with their respective references.
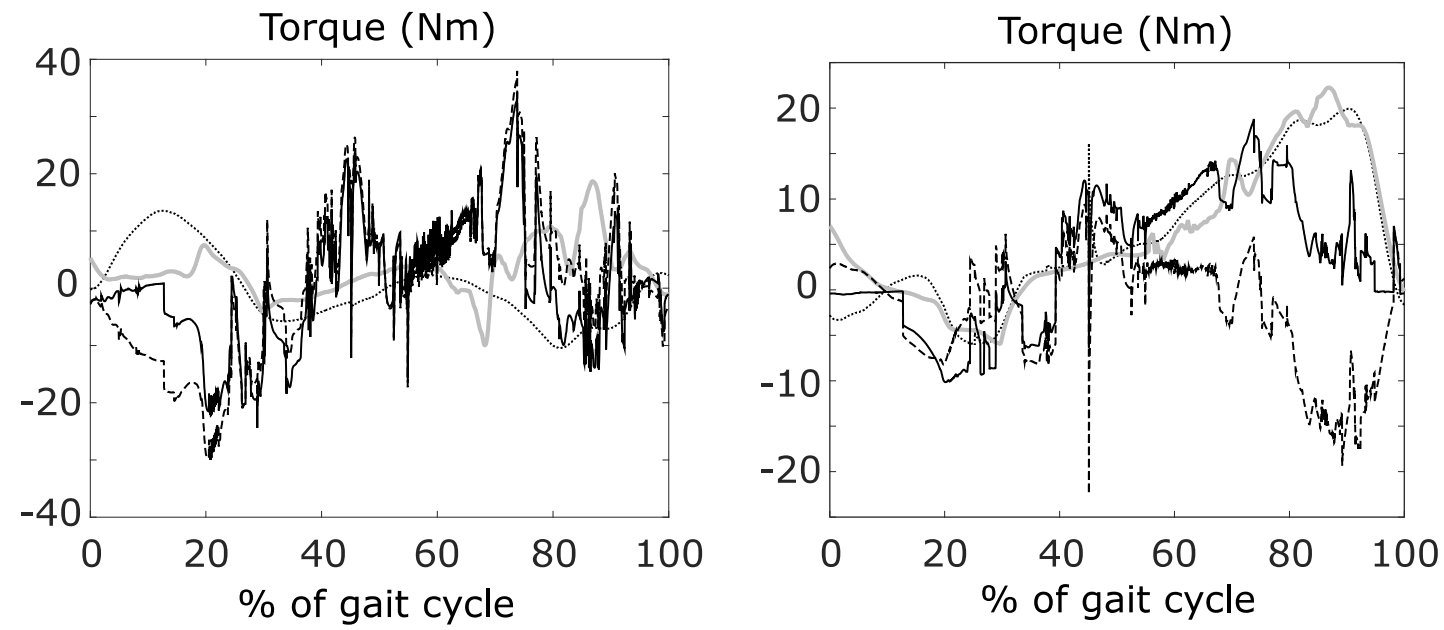

Figure 19. Torques in the right leg: knee level (left), ankle level (right). Torque exerted by orthosis (dotted), torque exerted by controller (dashed), total torque (addition of the former two, black solid), torque obtained from IDA (reference, grey solid).

\subsection{Orthosis model as independent body}

In the second option, the orthoses were considered as independent bodies (see Fig. 20). The lower link of each orthosis was connected to the subject's ankle by a revolute joint in the direction of the ankle axis, obtained upon processing of the acquired motion, while the upper link was connected to the lower link by another revolute joint at knee level, so 
that each orthosis added two degrees of freedom. Inertial properties were assigned to the corresponding bodies. Moreover, torsional spring-damper elements were included in the revolute joints at knee and ankle levels to represent the locking and anti-foot-drop mechanisms, respectively. The limb/orthosis connecting elements were modeled by linear spring-dampers linking points of the limb and the orthosis, both at hip and knee levels.

Additional natural coordinates were required this time to include the new four bodies in the 3D human model of sub-section 7.1. As illustrated in Fig. 20, for each orthosis one new point was defined at knee level (p1) and another one at hip level (p2), while the point at ankle level was simply shared with that already defined in the joint of the human model. Moreover, three new unit vectors were required for each part: v1 (shared with the foot) in the direction of the orthotic ankle axis, v2 and v3 for the lower link; v4 (shared with the lower link) in the direction of the orthotic knee axis for the upper link. Unit vector v1 is calculated from the acquired motion, as it is not known a priori. Also, it must be noted that the orthotic ankle and knee axes can be different, and in fact they were in the case addressed, so that v1 and v4 might not be the same unit vector. Finally, the relative angles between foot and lower link, and between lower and upper links, respectively, were also added to the list (they are not drawn in Fig. 20 due to the lack of space). Therefore, a total increment of four points, twelve unit vectors and four angles was registered for the two devices, so that the resulting 3D human model including orthoses as independent bodies and crutches possessed 61 degrees of freedom and was defined by a total of 298 mixed (natural and angular) dependent coordinates. The configuration vector $\mathbf{z}$ of 61 independent coordinates was formed by the three Cartesian coordinates of the lumbar joint plus the 18 sets of three angles defining the orientation of the bodies (Fig. 1), plus the two sets of two relative angles locating each orthotic open chain starting at the corresponding subject's ankle.

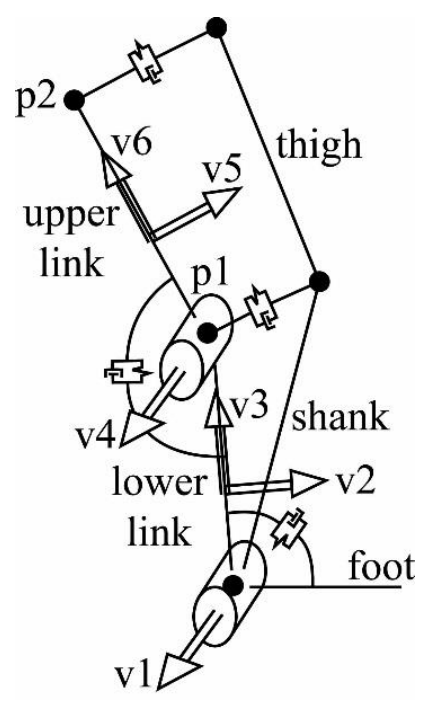

Figure 20. Modeling of the orthoses as independent bodies: additional natural coordinates (points and unit vectors) required.

\subsubsection{Fully actuated system}

The first approach to carry out the FDA of the acquired gait motion consisted of using trajectory tracking controllers (the CTC algorithm explained in sub-section 5.3) 
associated to all the subject's degrees of freedom. However, it must be noted that no control was exerted over the degrees of freedom of the orthoses.

Eq. (8), but limited this time to the subject's degrees of freedom, was used to calculate the required inputs along the simulation, assigning to the gains in matrix $\mathbf{C}_{\mathrm{P}}$ a value of $10^{3}$ and obtaining the gains in matrix $\mathbf{C}_{\mathrm{D}}$ by application of (9), yielding a value of 63.25. The simulation was run with a constant time step of $1 \mathrm{~ms}$.

The obtained results were similar in accuracy to those obtained for the case when the orthoses were modeled as embedded in the subject's legs (sub-section 7.1.1). However, this time histories of limb/orthosis misalignments and interaction forces could be calculated too.

Figure 21 shows the histories of the torques exerted by the right orthosis at knee and ankle levels. It can be seen that the addition of the torques exerted by the orthosis and the controller does not exactly match the torque obtained from IDA for the model with orthoses embedded in the legs (as it happened in sub-section 7.1.1), since now misalignment can exist between leg and orthosis.
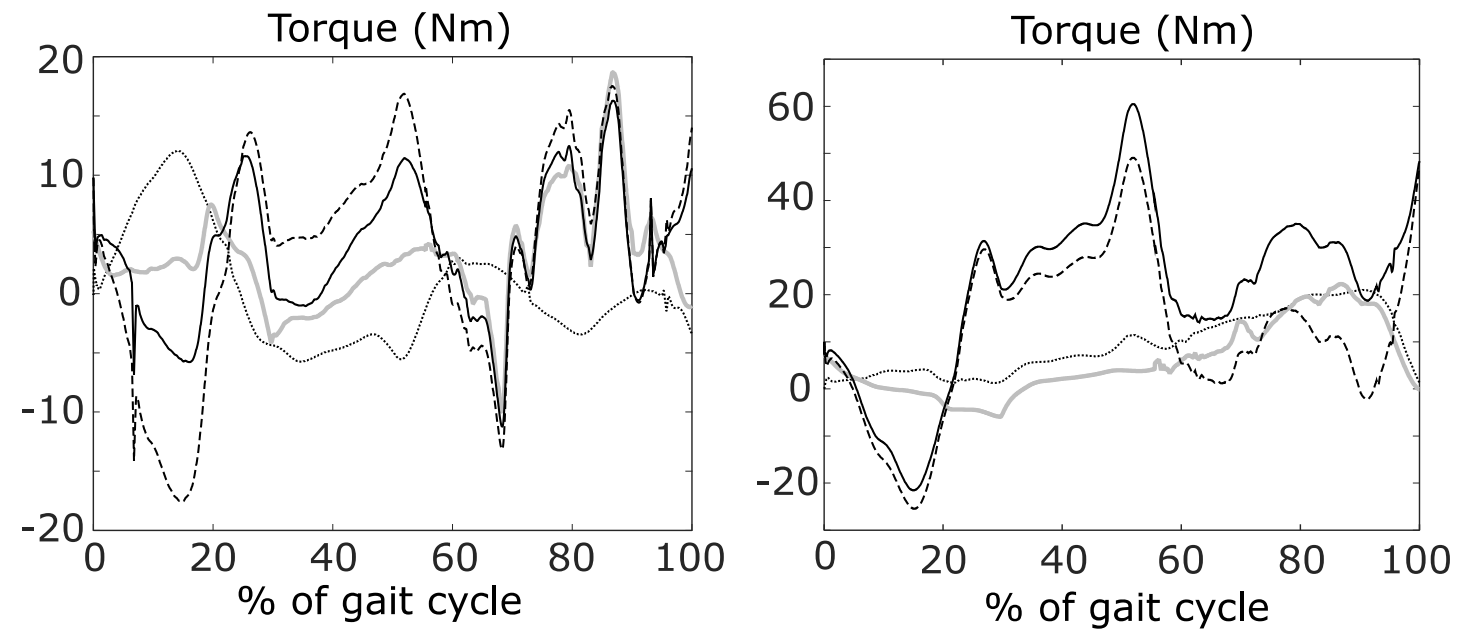

Figure 21. Torques in the right leg: knee level (left), ankle level (right). Torque exerted by orthosis (dotted), torque exerted by controller (dashed), total torque (addition of the former two, black solid), torque obtained from IDA (reference, grey solid).

Figure 22 shows the histories of the misalignments and the interaction forces between limb and orthosis, both at knee and hip levels. It can be seen that they are notably higher on the left side, thus indicating a strong asymmetry of the gait. Furthermore, while misaligment is higher at hip than at knee level for the left side, force interaction works the other way around. However, a different behavior is found on the right side, where misalignment and force interaction are more similar at both levels, and there is some kind of proportionality between both magnitudes. 

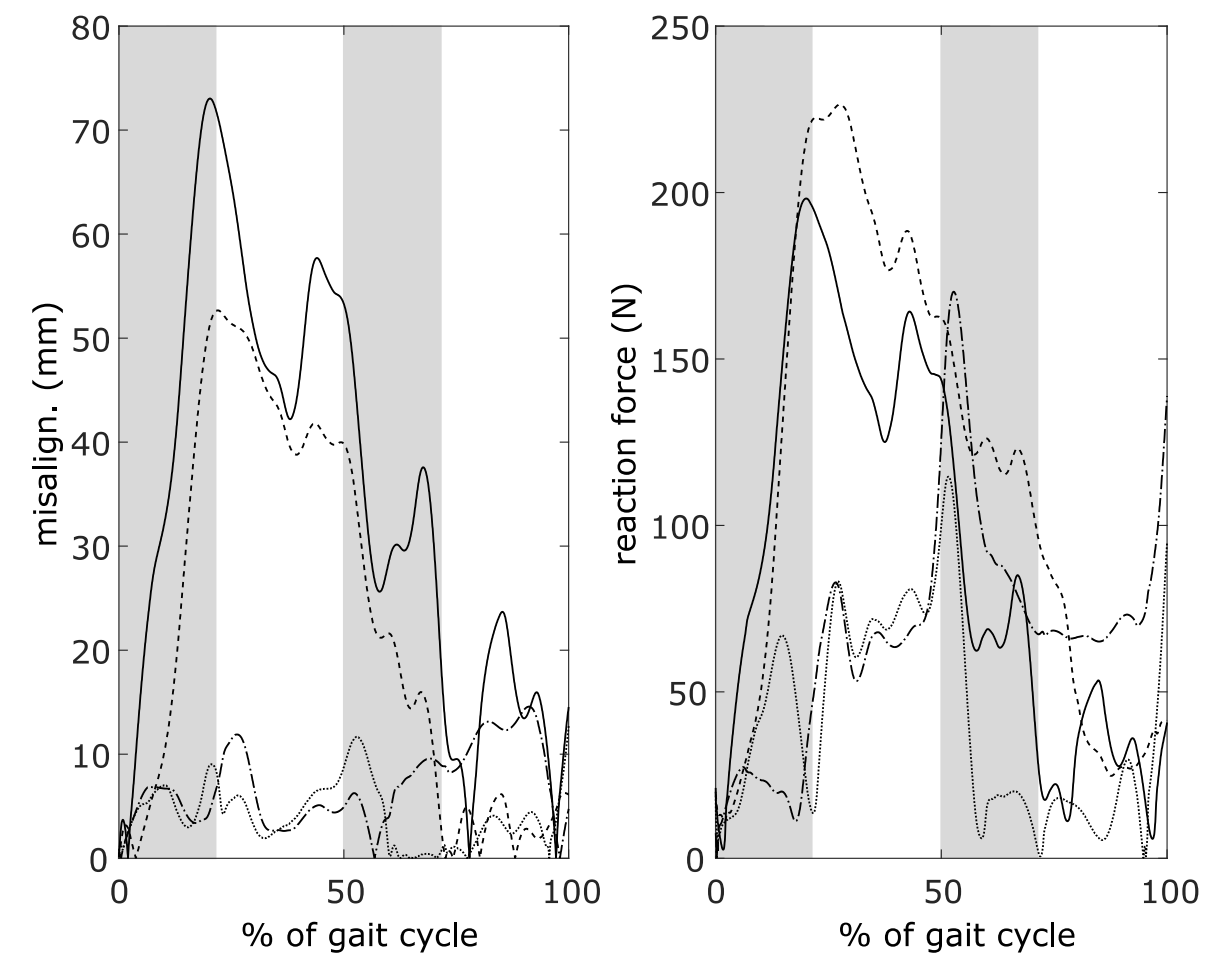

Figure 22. Limb/orthosis misalignment (left) and interaction force (right): left hip level (solid), right hip level (dotted), left knee level (dashed), right knee level (dasheddotted). Grey areas correspond to swing phases of right (first) and left foot.

\subsubsection{Underactuated system}

The second approach to carry out the FDA of the acquired gait motion was to consider the system as underactuated, i.e. with controllers acting at the subject's joints only, but not on the base body (Section 6). As in sub-section 7.2.1, the orthoses degrees of freedom were not controlled.

The contact models for the subject's feet and the crutches were the same used in subsection 7.1.2, i.e. when the orthoses were modeled as embedded in the subject's legs.

The control strategy adopted was the same applied in the 3D gait of a healthy subject in sub-section 6.3.2, i.e. to define more outputs than inputs, choosing as outputs the 57 coordinates of the subject's configuration vector $\mathbf{z}$. Therefore, the upper part of (16) was used to calculate the required inputs along the simulation, assigning to the gains in matrix $\mathbf{C}_{\mathrm{P}}$ a value of $10^{3}$ and obtaining the gains in matrix $\mathbf{C}_{\mathrm{D}}$ by application of (9), yielding a value of 63.25. The weights for the outputs needed to build weight matrix $\mathbf{W}$ were set to 1 . The time step used to run the simulation was $1 \mathrm{~ms}$. 

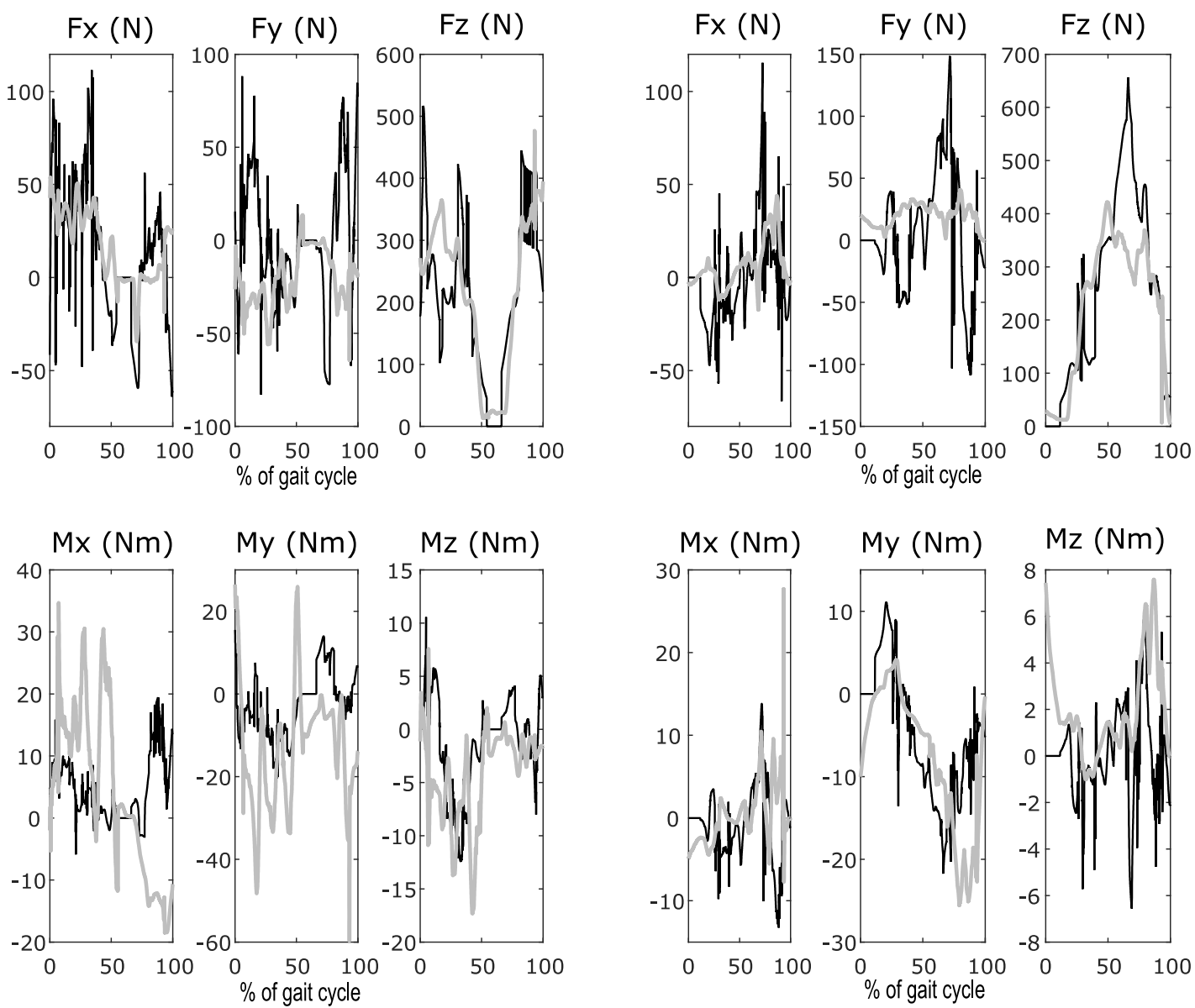

Figure 23. Comparison of contact reactions (forces and moments) obtained from forward dynamic simulation (black) and from IDA (grey) for the right foot (right) and left foot (left).

Again, as it happened for the case when the orthoses were modeled as embedded in the subject's legs (sub-section 7.1.2), the foot-ground and crutch-ground contact models obtained through optimization were directly usable in forward dynamics, yielding a good trajectory tracking without the necessity of any hand-made tuning. Also again, notable discrepancies were found between the ground reactions generated when the model was run in forward dynamics and those obtained from the IDA, as illustrated in Fig. 23. Despite this, the controllers managed to follow the acquired motion without falling, as illustrated in Fig. 24, with RMS errors of $0.023 \mathrm{~m}$ for the translational coordinates $(x, y$, $z$ of the lumbar joint), $5.2 \cdot 10^{-3} \mathrm{rad}$ for the angular coordinates, $48.38 \mathrm{~N}$ for the force components of the external reaction, $11.12 \mathrm{Nm}$ for the moment components of the external reaction and $27.24 \mathrm{Nm}$ for the net joint torques. 

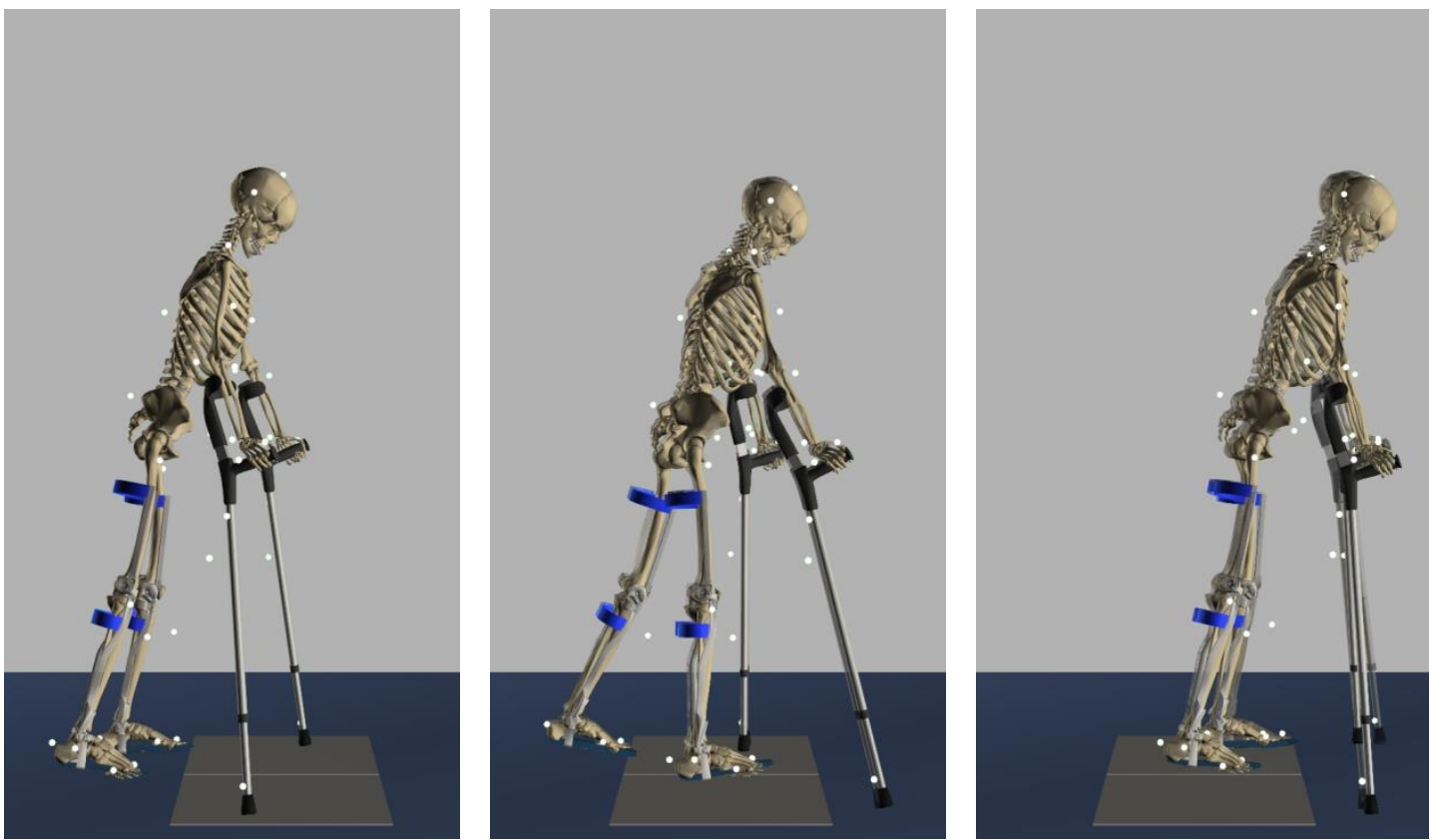

Figure 24. Postures of the model at the beginning (left), half (center) and end (right) of the forward-dynamics simulation compared with those of the acquired motion. In the three pictures, the two superimposed images correspond to the same time point.

Figure 25 shows the histories of the torques exerted by the right orthosis at knee and ankle levels. While results at ankle level are very similar to those obtained in the previous subsection (7.2.1), although a little bit noisier, the torque exerted by the controller exhibits more extreme values at knee level, which translates to the total torque. Again, as it happened in sub-section 7.2.1, the addition of the torques exerted by the orthosis and the controller does not match the torque obtained from IDA for the model with orthoses embedded in the legs due to the misalignment that may exist between leg and orthosis.
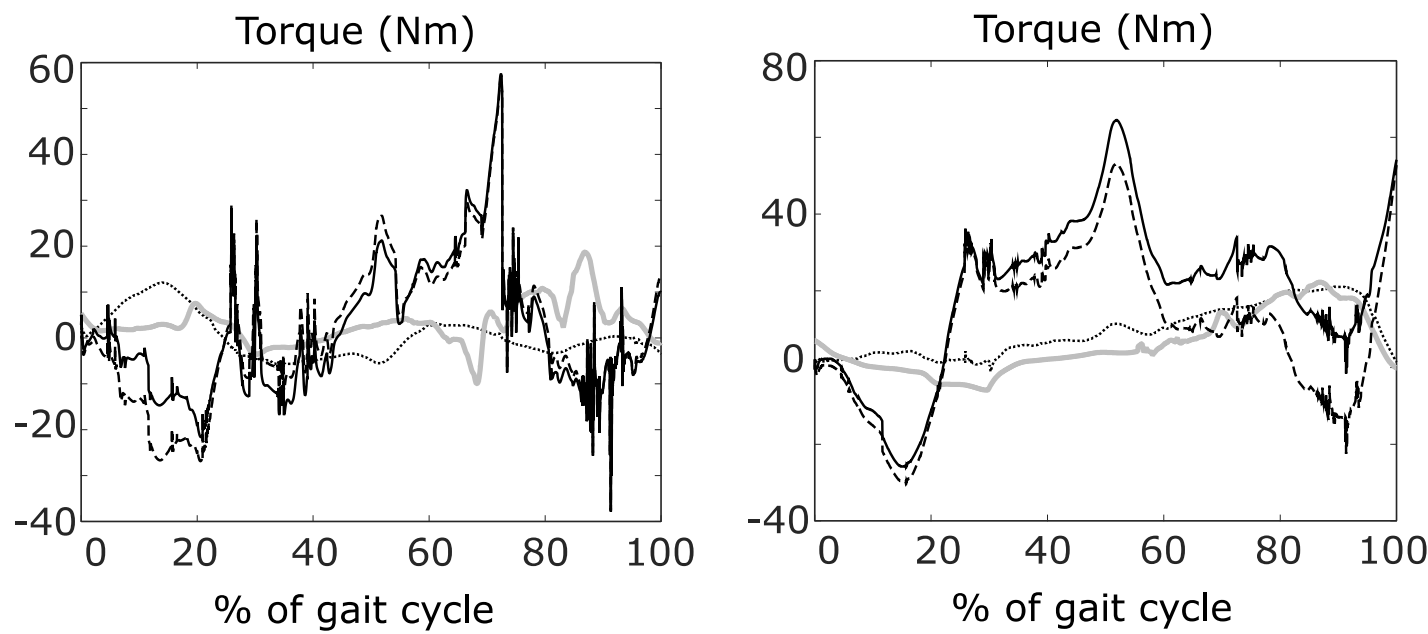

Figure 25. Torques in the right leg: knee level (left), ankle level (right). Torque exerted by orthosis (dotted), torque exerted by controller (dashed), total torque (addition of the former two, black solid), torque obtained from IDA (reference, grey solid). 
Figure 26 shows the histories of the misalignments and the interaction forces between

limb and orthosis, both at knee and hip levels. It can be seen that the plots are very similar to those obtained in sub-section 7.2.1.
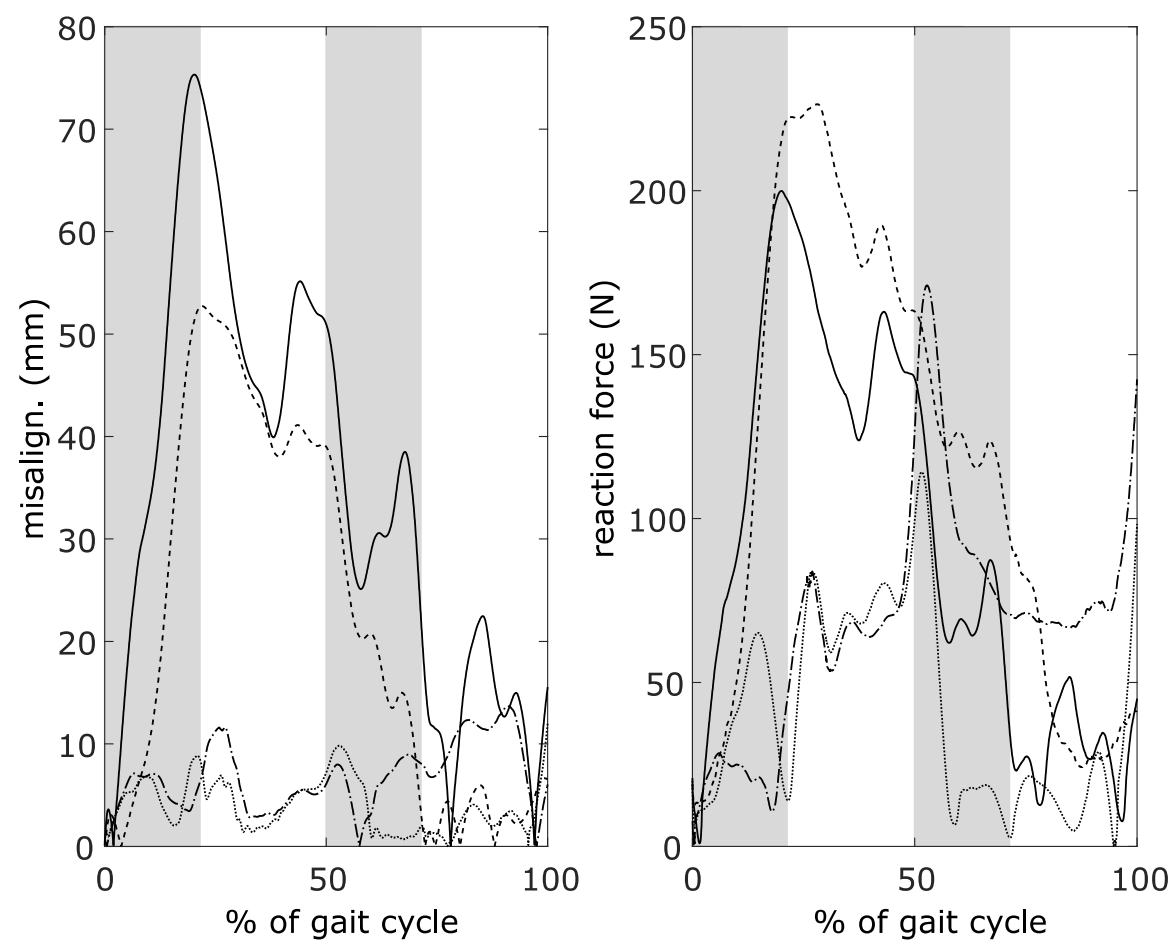

Figure 26. Limb/orthosis misalignment (left) and interaction force (right): left hip level (solid), right hip level (dotted), left knee level (dashed), right knee level (dasheddotted). Grey areas correspond to swing phases of right (first) and left foot.

\section{Discussion and conclusions.}

Sections 5-7 have addressed the forward-dynamics analysis of human gait at skeletal level by means of control methods: full actuation was applied in Section 5, using three control methods; underactuation was considered in Section 6, with several strategies in the selection of the outputs to be tracked, and in the way the ground reactions were included; both previous approaches were tested in Section 7 for the case of a gait assisted by orthoses and crutches, with two options for modeling the orthoses. Most relevant results are summarized in Table 4, where values are approximated to streamline comparison. 
Table 4. Summary of results of Sections 5 (full actuation), 6 (underactuation) and 7 (full actuation and underactuation applied to assisted gait). Time step of integration $1 \mathrm{~ms}$.

\begin{tabular}{|c|c|c|c|c|c|c|c|c|}
\hline Sec. & $\begin{array}{l}\text { Type } \\
\text { of gait }\end{array}$ & $\begin{array}{c}\text { Control } \\
\text { actuation }\end{array}$ & $\begin{array}{l}\text { RMSE } \\
\text { trans. } \\
\text { coord. }\end{array}$ & $\begin{array}{c}\text { RMSE } \\
\text { ang. } \\
\text { coord. }\end{array}$ & $\begin{array}{l}\text { RMSE } \\
\text { react. } \\
\text { forces }\end{array}$ & $\begin{array}{l}\text { RMSE } \\
\text { react. } \\
\text { mom. }\end{array}$ & $\begin{array}{l}\text { RMSE } \\
\text { drive } \\
\text { torq. }\end{array}$ & Remarks \\
\hline \multirow{3}{*}{5} & \multirow{3}{*}{ normal } & \multirow{3}{*}{ full } & failed & failed & failed & failed & failed & $\begin{array}{c}\text { computed } \\
\text { feedforward }\end{array}$ \\
\hline & & & 1.e-7 & 1.e-5 & 1.e-2 & 1.e-4 & 1.e-4 & $\begin{array}{l}\text { PD with } \\
\text { comp. f.f. }\end{array}$ \\
\hline & & & 1.e-6 & 1.e-4 & 1.e0 & 1.e-1 & 1.e-1 & CTC \\
\hline \multirow{2}{*}{6} & \multirow{2}{*}{ normal } & \multirow{2}{*}{ underact. } & 6.e-4 & 8.e-4 & $0 . \mathrm{e} 0$ & $0 . \mathrm{e} 0$ & 1.e-3 & $\begin{array}{l}\text { ext. react. } \\
\text { from IDA }\end{array}$ \\
\hline & & & 4.e-2 & 1.e-2 & 1.e2 & 1.e1 & 1.e1 & $\begin{array}{l}\text { ext. react. } \\
\text { cont. model }\end{array}$ \\
\hline \multirow{4}{*}{7} & \multirow{4}{*}{ assisted } & \multirow{2}{*}{ full } & 1.e-6 & 1.e-4 & 1.e0 & 1.e-1 & 1.e-1 & $\begin{array}{l}\text { orthoses } \\
\text { embedded }\end{array}$ \\
\hline & & & 1.e-6 & 1.e-4 & 1.e0 & 1.e-1 & 1.e-1 & $\begin{array}{l}\text { orthoses as } \\
\text { indep. bod. }\end{array}$ \\
\hline & & \multirow{2}{*}{ underact. } & 1.e-2 & 4.e-3 & 4.e1 & 1.e1 & 1.e1 & $\begin{array}{l}\text { orthoses } \\
\text { embedded }\end{array}$ \\
\hline & & & 2.e-2 & 5.e-3 & 4.e1 & 1.e1 & 2.e1 & $\begin{array}{l}\text { orthoses as } \\
\text { indep. bod. }\end{array}$ \\
\hline
\end{tabular}

From the obtained results (Section 5), it can be concluded that the CTC is a useful method to carry out the forward-dynamics analysis of an acquired gait motion at skeletal level, since it provides a good level of accuracy in both motion and external reactions, and requires almost no effort in tuning the gains of the controllers. Although, apparently, the approach has no advantages over an inverse-dynamics analysis for healthy gait at skeletal level, it can be exploited when descending to muscular level, as in that case the simultaneous integration of the equations of motion and the equations of muscular activation and contraction does provide better dynamic consistency than the inversedynamics approach.

Regarding the control strategy (Section 6), full actuation (i.e. actuation on all the subject's degrees of freedom) proved to be clearly superior to underactuation (i.e. actuation on the subject's joints only), although the underactuated case with input of the external reactions calculated from IDA yielded quite satisfactory results. When the external reactions come from a contact model, acceptable accuracy can be achieved at the configuration level only, while high errors appear at the force level since fitting the contact model revealed certainly problematic; higher resolution of the motion capture equipment could improve that (particularly critical for the feet), but it is not clear up to what extent [23]. It is true that the underactuated control strategies tested in this work were limited to the tracking of coordinates and ground reactions [21], and that perhaps other alternatives could be more successful: exploration of recent dynamic walking control methods for the gait of humanoid robots $[27,28]$ or virtual characters $[29,30]$ might be interesting in this sense.

Perhaps the most meaningful application of the forward-dynamics analysis of an acquired gait at skeletal level is the estimation of the interaction behavior between the subject's body and assistive devices (Section 7), which can hardly be obtained through an inversedynamics approach when the motion capture process doesn't provide enough resolution 
to distinguish the motions of limb and device. It has been shown that, in the case of lowerlimb orthotic devices, maximum information about the interactive behavior can be extracted when they are modeled as independent bodies, although additional work is required in this direction, as identification of the interface parameters and experimental validation of the results.

\section{Compliance with Ethical Standards}

Research involving human participants: This study was approved by the institutional ethical committee.

Informed consent: All participants in the experiments gave their informed consent.

Funding: This study was funded by the Spanish Ministry of Economy and Competitiveness (MINECO) under project DPI2015-65959-C3-1-R, cofinanced by the European Union through EFRD program.

Conflict of interest: J. Cuadrado is a member of the Editorial Board of the journal Multibody System Dynamics. The remaining authors have no conflict of interest.

\section{References}

[1] D.A. Winter, Biomechanics and motor control of human movement, $3^{\text {rd }}$ ed., Wiley, 2005.

[2] K.A. Abdel-Malek, J.S. Arora, Human motion simulation, Academic Press, 2013.

[3] R. Riemer, E.T. Hsiao-Wecksler, Improving net joint torque calculations through a two-step optimization method for estimating body segment parameters, Journal of Biomechanical Engineering, vol. 131, no. 1, pp. 011007-1-011007-7, 2009.

[4] L.J. Holmberg, A. Klarbring, Muscle decomposition and recruitment criteria influence muscle force estimates, Multibody System Dynamics, vol. 28, no. 3, pp. 283-289, 2012.

[5] D.G. Thelen, F.C. Anderson, Using computed muscle control to generate forward dynamic simulations of human walking from experimental data, Journal of Biomechanics, vol. 39, pp. 1107-1115, 2006.

[6] F. Michaud, U. Lugris, Y. Ou, J. Cuadrado, A. Kecskemethy, Influence of muscle recruitment criteria on joint reaction forces during human gait, ECCOMAS Thematic Conference on Multibody Dynamics, Barcelona, Spain, 2015.

[7] C. Quental, J. Folgado, J. Ambrosio, A window moving inverse dynamics optimization for biomechanics of motion, Multibody System Dynamics, vol. 38, no. 2, pp. 157-171, 2016.

[8] C. Quental, M. Azevedo, J. Ambrosio, S.B. Gonçalves, J. Folgado, Influence of the musculotendon dynamics on the muscle force sharing problem of the shoulder: a fully inverse dynamics approach, ASME Journal of Biomedical Engineering, vol. 140, no. 7, pp. 071005-1-071005-11, 2018.

[9] M. Ackermann, W. Schiehlen, Physiological methods to solve the force-sharing problem in biomechanics, C.L. Bottasso (Ed.) Multibody Dynamics Computational Methods and Applications, pp. 1-24, Springer, 2009.

[10] P.C. Silva, M.T. Silva, J.M. Martins, Evaluation of the contact forces developed in the lower limb/orthosis interface for comfort design, Multibody System Dynamics, vol. 24, no. 3, pp. 367-388, 2010. 
[11] H. Dallali, M. Mosadeghzad, G.A. Medrano-Cerda, N. Docquier, P. Kormushev, N. Tsagarakis, Z. Li, D. Caldwell, Development of a dynamic simulator for a compliant humanoid robot based on a symbolic multibody approach, IEEE Int. Conference on Mechatronics (ICM), Vicenza, Italy, 2013.

[12] M. Ackermann, A.J. van den Bogert, Optimality principles for model-based prediction of human gait, Journal of Biomechanics, vol. 43, no. 6, pp. 1055-1060, 2010.

[13] C.L. Vaughan, B.L. Davis, J.C. O’Connor, Dynamics of Human Gait, 2nd ed., Cape Town, Kiboho Publishers, 1999.

[14] J.A.C. Ambrosio, A. Kecskemethy, Multibody dynamics of biomechanical models for human motion via optimization, J.C. Garcia Orden, J.M. Goicolea, J. Cuadrado (Eds.) Multibody Dynamics - Computational Methods and Applications, pp. 245270, Springer, 2007.

[15] U. Lugris, J. Carlin, A. Luaces, J. Cuadrado, Gait analysis system for spinal cord injured subjects assisted by active orthoses and crutches, Journal of Multi-body Dynamics, vol. 227, no. 4, pp. 363-374, 2013.

[16] https://www.iftomm-multibody.org/benchmark/, Library of Computational Benchmark Problems (problem Gait 2D).

[17] J. Garcia de Jalon, E. Bayo, Kinematic and Dynamic Simulation of Multibody Systems, New York, Springer-Verlag, 1994.

[18] K.C. Gupta, Mechanics and Control of Robots, New York, Springer-Verlag, 1997.

[19] R. Seifried, Integrated mechanical and control design of underactuated multibody systems, Nonlinear Dynamics, vol. 67, pp. 1539-1557, 2012.

[20] W. Blajer, The use of servo-constraints in the inverse dynamics analysis of underactuated multibody systems, Journal of Computational and Nonlinear Dynamics, vol. 9, no. 4, pp. 041008-1-041008-11, 2014.

[21] A. Seth, M.G. Pandy, A neuromusculoskeletal tracking method for estimating individual muscle forces in human movement, Journal of Biomechanics, vol. 40, pp. 356-366, 2007.

[22] U. Lugris, J. Carlin, R. Pamies-Vila, J.M. Font-Llagunes, J. Cuadrado, Solution methods for the double-support indeterminacy in human gait, Multibody System Dynamics, vol. 30, no. 3, pp. 247-263, 2013.

[23] M.S. Shourijeh, J. McPhee, Foot-ground contact modeling within human gait simulations: from Kelvin-Voigt to hyper-volumetric models, Multibody System Dynamics, vol. 35, no. 4, pp. 393-407, 2015.

[24] N. Hansen, The CMA evolution strategy: a comparing review, Studies in Fuzziness and Soft Computing, vol. 192, pp. 75-102, 2006.

[25] D. Dopico, A. Luaces, M. Gonzalez, J. Cuadrado, Dealing with multiple contacts in a human-in-the-loop application, Multibody System Dynamics, vol. 25, no. 2, pp. 167-183, 2011.

[26] P. Flores, M. Machado, M.T. Silva, J.M. Martins, On the continuous contact force model for soft materials in multibody dynamics, Multibody System Dynamics, vol. 25, no. 3, pp. 357-375, 2011.

[27] J. Englsberger, C. Ott, A. Albu-Schäffer, Three-dimensional bipedal walking control based on divergent component of motion, IEEE Transactions on Robotics, vol. 31, no. 2, pp. 355-368, 2015.

[28] R. Dehghani, A. Fattah, E. Abedi, Cyclic gait planning and control of a five-link biped robot with four actuators during single support and double support phases, Multibody System Dynamics, vol. 33, no.4, pp. 389-411, 2015. 
[29] J.M Wang, S.R. Hamner, S.L. Delp, V. Koltun, Optimizing locomotion controllers using biologically-based actuators and objectives, ACM Transactions on Graphics, vol. 31, no. 4, article no. 25, 2012.

[30] T. Geijtenbeek, M. van de Panne, A.F. van der Stappen, Flexible muscle-based locomotion for bipedal creatures, ACM Transactions on Graphics, vol. 32, no. 6, article no. 206, 2013. 